\title{
A Systematic Literature Review on BPMN Extensions
}

\author{
Karim Zarour ${ }^{1}$, Djamel Benmerzoug ${ }^{1}$, Nawal Guermouche ${ }^{2}$, Khalil Drira ${ }^{3}$ \\ ${ }^{1}$ Univ. Constantine, Algeria \\ 2 LAAS-CNRS, INSA, Univ. de Toulouse, France \\ ${ }^{3}$ LAAS-CNRS, CNRS, Univ. de Toulouse, France
}

\begin{abstract}
Despite the existence of several languages, BPMN has become the leading standard for business process modelling thanks to its expressiveness and semantic richness. However, BPMN is generic and still suffers from some limitations, which has prompted researchers to extend it, either for dealing with processes of specific domains like healthcare and manufacturing or for improving the language itself in terms of flexibility, variability, complexity, etc. This paper presents a systematic literature review that we conducted in order to determine the current state of the art of BPMN extensions. After the search and filtering of papers, 49 extensions were retained to be thoroughly examined and compared according to a set of criteria including objective, targeted domain, conformity to the extension mechanism, demonstration, implementation, etc. Based on the obtained results, we identified several gaps and suggested recommendations to fill them and advance the research field of extending BPMN.
\end{abstract}

Keywords: BPMN extension, Systematic literature review, Business process modelling, Modelling languages, Domain-specific business processes, Domain-specific modelling languages, OMG recommendations, MOF meta-model, XML Schema

\section{Introduction}

Given today's fiercely competitive and highly dynamic market, most modern companies are adopting a business process management (BPM) strategy, which ensures a continuous improvement of business processes (BPs) and their adaptation to change. BPM includes several activities such as automation, execution, and monitoring of BPs, but the modelling activity remains the most crucial one. Indeed, it allows specifying the way of carrying out BPs, which has a direct influence on the quality of a company's deliverables and thus on customer satisfaction. In addition, the errors and defects must be identified at the modelling phase where correction is cheaper. In fact, modelling is the first phase in the BP lifecycle and the cost of corrections increases exponentially over the lifecycle. Furthermore, BP models serve as a basis for knowledge sharing, quality of service, regulatory compliance, and stakeholder collaboration (Moreno-Montes de Oca et al., 2015).

Despite the existence of several modelling languages, Business Process Model and Notation (BPMN) has become the de-facto standard for BP modelling (Arevalo et al., 2016) (Yousfi et al., 2016) (Braun \& Esswein, 2014). In fact, BPMN is defined by the Object Management Group (OMG) and specified as ISO standard (ISO/IEC 19510:2013). The BPMN language is widely used for its expressiveness, simplicity and semantic richness. Moreover, it is supported by a wide range of tools like Activiti, jBPM, and Bizagi. However, BPMN becomes limited in supporting specific domains or non-functional properties since it provides generic elements and focuses only on the functional requirements of BPs.

In order to overcome this shortcoming and expand the use cases of BPMN, the OMG has introduced an extension mechanism allowing users to integrate new elements and provide valid BPMN extensions (OMG, 2013). Indeed, unlike the UML language that provides an extension mechanism by 
specialization (through UML profiles), the BPMN language allows extension by addition, which consists of attaching new domain-specific elements to the predefined elements of the language (OMG, 2013).

Furthermore, BP designers prefer extending BPMN and reusing its kernel to take advantage of its benefits (e.g., standardization, tool support) instead of developing a domain-specific modelling language (DSML) from the scratch, which is very costly and time-consuming (Braun and Esswein, 2014). Accordingly, a large and growing number of BPMN extensions are proposed in the literature targeting various objectives such as the representation of domain-specific BPs (e.g., healthcare, IOT, manufacturing) or the improvement of the BPMN language itself (e.g., flexibility, complexity, variability).

In this paper, we rely on guidelines depicted in (Kitchenham, 2007) to conduct a systematic literature review (SLR) that aims to determine the current state of the art of BPMN extensions and identify the gaps that should be filled in this research area. An SLR is distinguished from other types of literature review primarily by a comprehensive literature search and specification of research questions that should be addressed (Kitchenham, 2007). To the best of our knowledge, (Braun \& Esswein, 2014) is the only existing literature review that focuses on work extending the BPMN language. In fact, the authors have classified 30 extensions published between 2007 and 2014. However, the BPMN extension mechanism was introduced in 2011 and therefore previously published extensions cannot be judged on their conformance.

Our SLR complements that of (Braun \& Esswein, 2014) in terms of both literature and criteria. Indeed, after the search and filtering of papers, 49 BPMN extensions published after the last extension treated in (Braun \& Esswein, 2014) are retained for in-depth examination and comparison. To achieve this, we have established a set of criteria such as objective, target domain, representation format, conformance, implementation, evaluation, etc. Our study also aims to see how the characteristics of BPMN extensions have evolved over the past few years by comparing our results with those of (Braun \& Esswein, 2014).

The remainder of this paper is organized as follows. Section 2 overviews BP modelling techniques and in particular the BPMN language as well as its extension mechanism. Section 3 describes the methodology followed to conduct our SLR. In section 4, BPMN extensions are analysed and the obtained results are reported. Some related work are tackled in Section 5. Finally, Section 6 concludes this paper and provides directions for future work.

\section{Background}

In this section, we first highlight some process modelling techniques, and then we give an overview of the BPMN language as well as its extension mechanism.

\subsection{Business process modelling techniques}

Among the techniques that have been used for BP modelling, we can highlight flow diagrams, Petri nets, event driven process chain (EPC), and role activity diagrams. There are also some languages such as Business Process Modelling Language (BPML) that have appeared but soon discarded due to the lack of market acceptance. So far, UML and BPMN are the main standards.

It is worth mentioning that BPMN was not set for competing with existing BP languages like UML Activity Diagrams, BPEL, YAWL, and XPDL, but rather for complementing them. In fact, BPMN has been intended as an alternative language to be used by business analysts without technical knowledge, whereas UML was designed for software engineers, YAWL and BPEL provide respectively graphical and XML-based notations for executable processes, and XPDL was intended as a portable exchange format.

\subsection{Overview of the BPMN language}


The Business Process Model and Notation (BPMN) is a standard language for modelling BPs that is widely used in both academia and industry (Braun et al., 2016). BPMN is defined by the Object Management Group (OMG) and specified as ISO standard. BPMN in its latest release provides five basic categories of elements: flow objects, data, connecting objects, swimlanes, and artefacts (OMG, 2013). Based on the list of the core BPMN elements depicted in (OMG, 2013, p.26), we have created a metamodel (see figure 1) in which we structure BPMN elements according to their category.

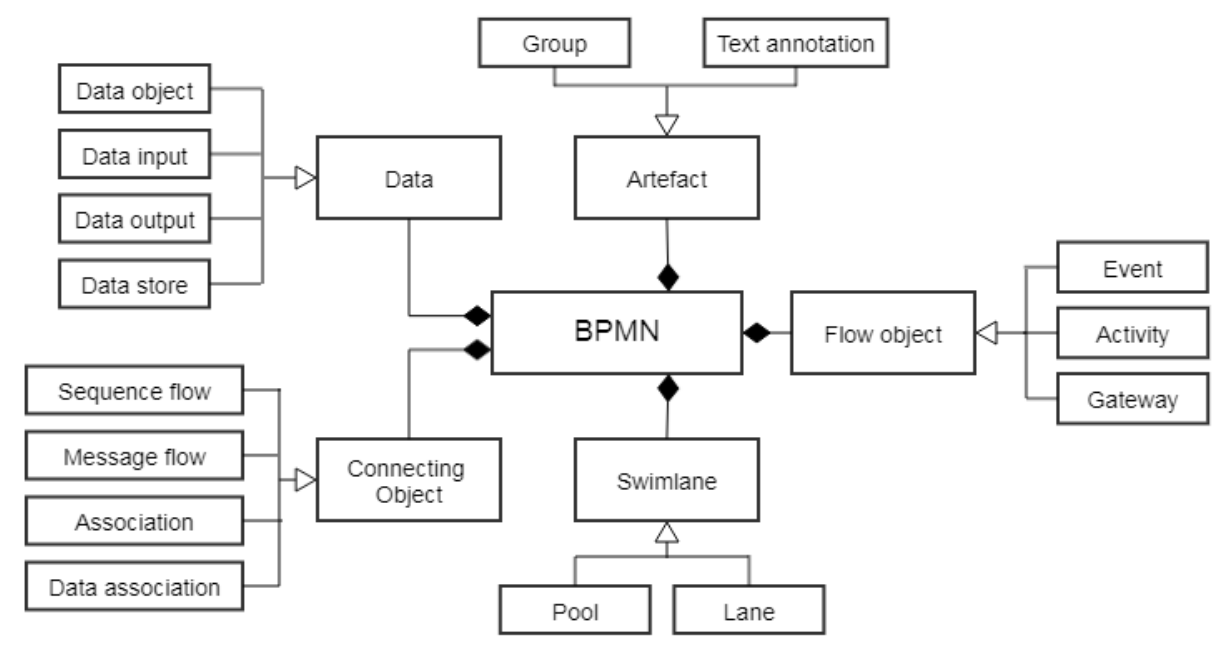

Figure 1. Structuring BPMN elements according to their category

BPMN is distinguished by a semantic richness, great expressiveness and ease of interpretation, thus reducing the risk of erroneous knowledge transfer (OMG, 2013). In addition, BPMN is supported by a wide range of modelling tools (e.g. Activiti, BPMN2 Modeler, Bizagi) and its models can be easily converted into executable languages (e.g., BPEL) (Pillat et al., 2015).

\subsection{BPMN extension mechanism}

BPMN 2.0.2 provides an extension mechanism that allows representing additional concepts and attaching them to its original elements in order to represent characteristics of a particular domain (e.g., health care, quality management, security, etc.). Extending BPMN and reusing its kernel allow taking advantage of its benefits (e.g., standardization, tool support) and avoiding expensive development of a domain-specific modelling language (DSML) from the scratch (Braun and Esswein, 2014).

For the specification of valid extensions, there are two representations defined in the official documentation of BPMN 2.0.2 (OMG, 2013). The first one is the meta-model represented in figure 2 and that is specified using the OMG's Meta Object Facility (MOF). This extension mechanism consists of four main elements (OMG, 2013):

1. Extension

2. ExtensionDefinition

3. ExtensionAttributeDefinition

4. ExtensionAttributeValue 


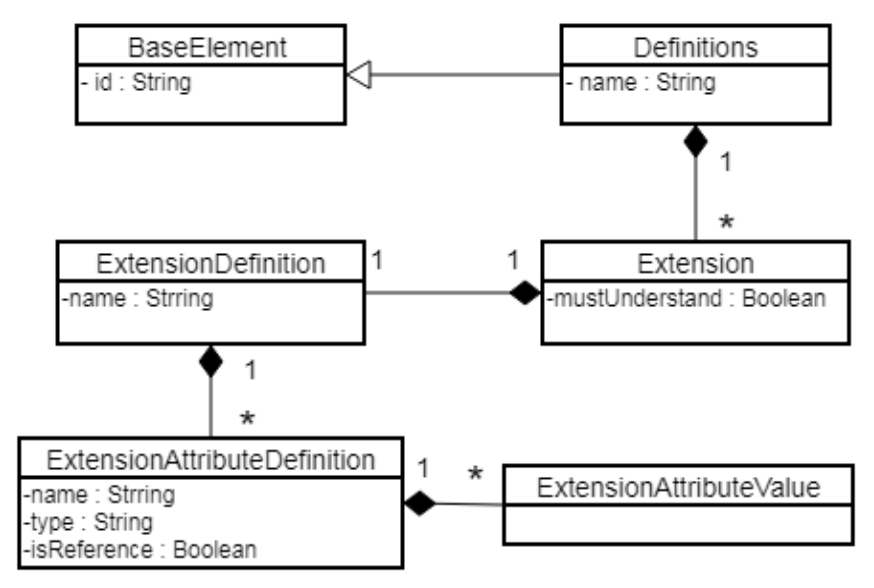

Figure 2. Extension class diagram (OMG, 2013)

Each BPMN element which subclasses the BPMN BaseElement can be extended by additional attributes. ExtensionDefinition groups new attributes under a new concept name and can be created independently of any BPMN definition. However, in order to use this meta-class to represent an extension, it must be associated with the meta-class Extension that binds an ExtensionDefinition and its attributes to the definition of a specific BPMN model (meta-class Definitions) (OMG, 2013).

An Extension Definition consists of several ExtensionAttributeDefinitions, which define the list of new attributes that can be attached to any BPMN element. The values of new attributes are stored in ExtensionAttributeValue meta-class (OMG, 2013). The mustUnderstand attribute indicates whether the semantics defined by the extension definition must be understood in order to process the BPMN model correctly. The default value is false. As for isReference attribute, it indicates if the attribute value will be referenced or contained (OMG, 2013).

The second format for representing valid BPMN extensions is a set of domain-specific elements represented in XML Schema that is saved into a BPMN file (i.e., a file with BPMN extension). The XML Schema representation specifies the interchange format for BPMN models (Pillat et al., 2015) and can support the definition of complex extensions that can be processed by BPMN tools. Nonetheless, the MOF-based extension mechanism has a limited capability. For instance, it does not define the type structures of the new attributes (OMG, 2013; Pillat et al., 2015).

The authors of (Stroppi et al., 2011) consider that the weak point in the BPMN extension mechanism is the lack of methodological guides for developing BPMN extensions. To fill this gap, they propose a procedure based on Model-Driven Architecture (MDA) for the methodical development of valid BPMN extensions. This method consists of four main steps (Stroppi et al., 2011):

1. Design of a Conceptual Domain Model of the Extension (CDME) using UML.

2. Definition of BPMN+X model describing an extension in terms of the BPMN extension mechanism. In this step, CDME elements are typed as 'BPMN concept' or 'Extension concept' using UML stereotypes.

3. Transformation of the BPMN+X model into an XML Schema Extension Definition Model.

4. Transformation of the XML Schema Extension Definition Model into an XML Schema Extension Definition Document.

In contrast, the Stroppi method has been criticized by (Braun \& Schlieter, 2014) for the lack of domain requirement analysis. In addition, it does not consider whether a domain concept needs to be integrated as a new element or BPMN is semantically sufficient to support it. Therefore, (Braun \& Schlieter, 2014) have extended the Stroppi method by a deep analysis of domain requirements and a comparison of domain-specific concepts with BPMN basic concepts. After defining the BPMN language 
and its extension mechanism, we will present in the following section the methodology followed to conduct the literature review.

\section{Methodology}

A Systematic Literature Review (SLR) is a specific type of literature reviews that is characterized by (Kitchenham, 2007):

- A specification of research questions that should be addressed

- A comprehensive and unbiased search for the relevant literature

- An explicit definition of inclusion and exclusion criteria

One of the main reasons for undertaking an SLR is to summarize and evaluate existing work in a given research area, identify their gaps, and suggest work to address them (Kitchenham, 2007). Based on the guidelines depicted in (Kitchenham, 2007), we conducted our SLR in several stages:

1. Formulating the research questions

2. Extracting and filtering papers

3. Defining evaluation and comparison criteria

4. Presenting and discussing the obtained results

The remainder of this section describes the details of each stage.

\subsection{Formulating the research questions}

The specification of research questions (RQs) is the most important part of any SLR as they guide authors throughout the review process (Kitchenham, 2007). The RQs that should be addressed in our SLR are formulated as follows:

RQ1: What are the areas and goals targeted by BPMN extensions these last years?

RQ2: What are the formats used for the representation of BPMN extensions?

RQ3: Do the proposed extensions comply with the extension mechanism specified by the OMG?

RQ4: How are BPMN extensions demonstrated, implemented, and evaluated?

\subsection{Extracting and filtering papers}

To retrieve papers proposing BPMN extensions, we constructed our search string firstly by combining the main terms 'BPMN' and 'Extension'. To make the search as comprehensive as possible and do not forget any BPMN extension, we replaced the term 'BPMN' with 'Modelling Language' and the term 'extension' was replaced by several derived words (e.g., 'Extending') or belonging to the same semantic field (e.g., 'Enhancement'). The final search string is structured as follows:

Search string = ("BPMN" OR "Modelling language" AND "Exten*" OR "Enhanc*" OR "Expan*" OR "Customiz" OR "Adapt").

We resorted to several databases and search engines like Web of Science, Scopus, ScienceDirect, Emerald, SpringerLink, ACM, DBLP, Google Scholar, AIS Digital Library, IEEE Xplore Digital Library, INSPEC, etc. Besides, each found article was used for a backward search through its related work section.

Our SLR targets all BPMN extensions published over the timespan of November 6, 2014 to December 24, 2018 in journals, conference/workshop proceedings, and book chapters. For this, we filtered the obtained papers according to the following exclusion criteria: 
- Papers that extend other modelling languages (e.g., UML, EPC) or execution languages like BPEL.

- Papers published before November 2014 whether or not they are treated in (classif Braun, 2014).

- Papers that are not published in journals, conference/workshop proceedings, and book chapters such as master and doctoral theses.

- Papers that do not propose a new BPMN extension.

- Papers written in a language other than English.

- Papers that describe the same BPMN extension in the same way.

We have found several papers in the literature that deal with similar BPMN extensions. For work where the extension is represented and implemented in the same way, only the most recent publication is retained. For example, the papers (Ben Hassen et al., 2017a), (Ben Hassen et al., 2017c), and (Ben Hassen et al., 2017b) present similar extensions in terms of objective, representation and implementation. Thus, only (Ben Hassen et al., 2017a) was selected. On the other hand, if the extensions are presented or implemented differently, they will both be considered as we did with (Yousfi et al., 2015) and (Yousfi et al., 2016) that share the same domain but the extensions are represented differently.

Filtering has greatly reduced the number of papers. In fact, after the paper collection, we obtained a total of 93 papers. Next, we discarded papers that were published before November 5, 2014 (including extensions treated in (Classif Braun, 2014)), duplicate/similar papers and those in which we did not find an extension of the BPMN language. However, we have kept the papers that propose an extension of BPMN whether it is primary or secondary contribution. Accordingly, a set of 49 papers were retained for an in-depth examination in our SLR. In figure 3, we used the BPMN language to model the main steps of paper extraction and filtering process.

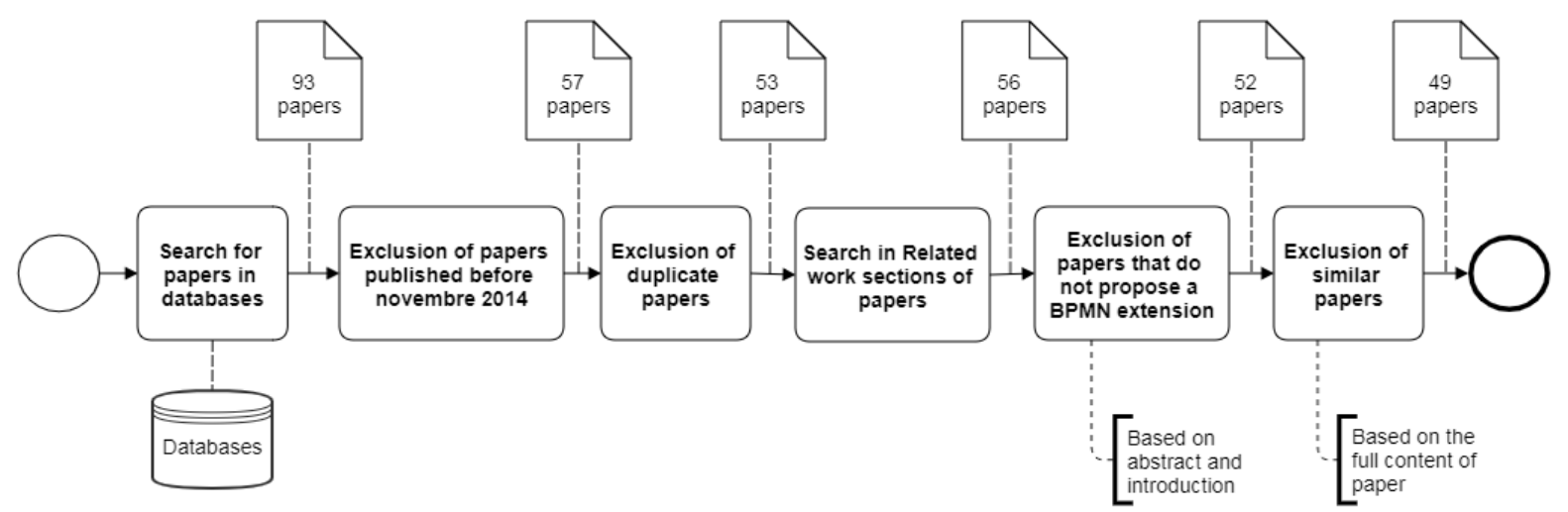

Figure 3. Process of paper extraction and filtering

\subsection{Defining evaluation and comparison criteria}

In order to evaluate and compare the BPMN extensions, we have defined the following criteria:

- Publication type: indicates if the extension has been published in a journal, a conference/workshop proceeding, or a book chapter.

- Aim: indicates the reason for which the extension was proposed or the problem that it solves.

- Category: we have defined two categories to classify the BPMN extensions according to their purpose. The first category 'Domain-specific BP' is for extensions intended to represent or handle the processes of a particular domain such as healthcare, manufacturing, Internet of Things (IoT), etc. The second category 'BP improvement' encompasses extensions that aim to improve the BPMN language (e.g., expressiveness, complexity, flexibility, variability), extensions that specify BP requirements in terms of different criteria (e.g., cost, performance 
, security, compliance, quality) and extensions that contribute to BPM activities (e.g., simulation, execution, monitoring, process mining). The extensions of the second category are independent of a specific domain (i.e., they can be used in any domain).

- BPMN version: specifies the extended version of the BPMN language (e.g., 1.2, 2.0, 2.0.2)

- Extension name: indicates whether a name has been assigned to the proposed extension.

- Main domain: Designates the main domain targeted by an extension knowing that some extensions deal with multiple domains (e.g., security in healthcare processes) but only the main domain is considered.

- Demonstration: indicates whether an extension has been demonstrated through an illustrative example and whether the provided example is concrete or abstract.

- Implementation: mentions whether a BPMN extension has been implemented either by integrating it into a modelling tool (e.g., plugin, code injection) or by developing a new tool.

- Evaluation: specifies for each extension whether it has been evaluated and which method is used for the evaluation (e.g., experimentation, use of metrics, comparison with other extensions, comparison with the BPMN language itself).

- Conformity: determines whether a BPMN extension complies with the OMG's recommendations. An extension is considered conform if it is represented through a metamodel or an XML schema defined in the official documentation of the BPMN language. We also consider that the methods of (Stroppi et al., 2011) and (Braun \& Schlieter, 2014) are conform because they are based on the MOF-meta model by specifying the correspondences to its meta-classes through stereotypes.

- Representation: mentions which of the three formats (Meta-model, XML Schema, and graphical elements) are used for the representation of the proposed extension.

- Affected BPMN elements: lists the BPMN elements that have been affected by the extension. A BPMN element is considered affected if it is reused, customized, or extended.

\subsection{Presentation and discussion of the results}

This last step is devoted to the presentation, interpretation, and analysis of the results obtained after a deep examination of each paper. For this, we begin by classifying, comparing, and assessing all BPMN extensions in tables according to the criteria defined in the previous stage. Then we give some statistics using graphs like pie charts and histograms. Finally, we interpret the obtained results and we provide explanations.

\section{Results of the review}

In table 1, we define for each BPMN extension the publication type ('J' for journal, ' $C$ ' for conference or workshop and ' $\mathrm{Ch}$ ' for chapter), the main purpose of the extension as well as the category ('Imp' 'for $B P$ improvement and 'DS' for domain-specific BP).

Table 1. Comparison of BPMN extensions according to their publication type, aim, and category

\begin{tabular}{|c|c|c|c|}
\hline 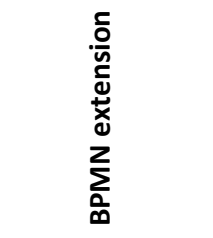 & 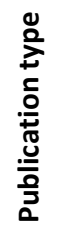 & Extension aim & 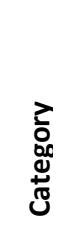 \\
\hline $\begin{array}{l}\text { (Anseeuw \& al., } \\
\text { 2015) }\end{array}$ & $\mathrm{C}$ & Support modelling and monitoring of decentralized BPs & DS \\
\hline $\begin{array}{l}\text { (Arevalo et al., } \\
\text { 2016) }\end{array}$ & J & $\begin{array}{l}\text { Integrate time aspects within BP models such as temporal dependencies between } \\
\text { activities and deadlines }\end{array}$ & Imp \\
\hline $\begin{array}{l}\text { (Ben Hassen et } \\
\text { al., 2017a) }\end{array}$ & J & Include crucial knowledge created and mobilized by sensitive BPs & Imp \\
\hline
\end{tabular}




\begin{tabular}{|c|c|c|c|}
\hline $\begin{array}{l}\text { (Ben said et al., } \\
\text { 2017) }\end{array}$ & $\mathrm{C}$ & $\begin{array}{l}\text { Deal with the flexibility of Inter-organizational BPs modelled through a version-based } \\
\text { approach }\end{array}$ & $\operatorname{Imp}$ \\
\hline $\begin{array}{l}\text { (Betke \& Seifert, } \\
\text { 2017) }\end{array}$ & $\mathrm{C}$ & Specify the requirements of disaster response processes & DS \\
\hline $\begin{array}{l}\text { (Bocciarelli et al., } \\
\text { 2017a) }\end{array}$ & $\mathrm{C}$ & Represent cyber-physical systems as resources supporting BP execution & DS \\
\hline $\begin{array}{l}\text { (Bocciarelli \& al., } \\
\text { 2017b) }\end{array}$ & $\mathrm{C}$ & Define structures of data exchanged during the execution of BP collaborations & $\operatorname{Imp}$ \\
\hline $\begin{array}{l}\text { (Bocciarelli et al., } \\
\text { 2016) }\end{array}$ & $\mathrm{C}$ & Enable the dynamic allocation of resources to BP tasks & $\operatorname{Imp}$ \\
\hline $\begin{array}{l}\text { (Braun \& Esswein, } \\
\text { 2015) }\end{array}$ & $\mathrm{C}$ & Reduce BPMN complexity by providing appropriate views on BP models & Imp \\
\hline $\begin{array}{l}\text { (Braun et al., } \\
\text { 2015) }\end{array}$ & $\mathrm{C}$ & $\begin{array}{l}\text { Model healthcare processes that involve different stakeholders such as physicians } \\
\text { and nurses }\end{array}$ & DS \\
\hline $\begin{array}{l}\text { (Braun et al., } \\
\text { 2016) }\end{array}$ & $\mathrm{C}$ & Represent resources and documents used in clinical pathways & DS \\
\hline $\begin{array}{l}\text { (Cartelli et al., } \\
\text { 2016) }\end{array}$ & $\mathrm{C}$ & $\begin{array}{l}\text { Represent resources and external factors that may affect the process execution under } \\
\text { a cost-sensitive perspective }\end{array}$ & $\operatorname{Imp}$ \\
\hline $\begin{array}{l}\text { (Carvalho et al., } \\
\text { 2018) }\end{array}$ & $\mathrm{C}$ & $\begin{array}{l}\text { Provide an aspect-oriented BP modelling notation to enhance the readability and } \\
\text { simplicity of BPMN models }\end{array}$ & $\operatorname{Imp}$ \\
\hline $\begin{array}{l}\text { (Chergui \& } \\
\text { Benslimane, } \\
\text { 2018) }\end{array}$ & $\mathrm{C}$ & Specify cyber security requirements and improve the system's security analysis & DS \\
\hline $\begin{array}{l}\text { (Chiu \& Wang, } \\
\text { 2015) }\end{array}$ & $\mathrm{C}$ & Introduce new types of events to take into account loT aspects in process modelling & DS \\
\hline $\begin{array}{l}\text { (D’Ambrogio et } \\
\text { al., 2016) }\end{array}$ & $\mathrm{C}$ & Annotate BP models with performance requirements and simulations results & $\operatorname{Imp}$ \\
\hline $\begin{array}{l}\text { (De Giacomo et } \\
\text { al., 2015) }\end{array}$ & $\mathrm{C}$ & Add declarative constructs to BPMN for providing a hybrid process modelling & Imp \\
\hline $\begin{array}{l}\text { (Domingos et al., } \\
\text { 2016) }\end{array}$ & $\mathrm{Ch}$ & $\begin{array}{l}\text { Register reliability informations to reduce the failure rate of loT-aware BPMN } \\
\text { healthcare processes }\end{array}$ & DS \\
\hline $\begin{array}{l}\text { (Dorndorfer \& } \\
\text { Seel, 2017) }\end{array}$ & $\mathrm{C}$ & $\begin{array}{l}\text { Enable the modelling of mobile context informations and their influence on sensitive } \\
\text { BPs }\end{array}$ & DS \\
\hline $\begin{array}{l}\text { (Dukaric \& Juric, } \\
\text { 2018) }\end{array}$ & J & Orchestrate Cloud-specific workflow activities in BP engines & DS \\
\hline $\begin{array}{l}\text { (Graja et al., } \\
\text { 2016) } \\
\text { implemented in } \\
\text { (Graja et al., } \\
\text { 2017) }\end{array}$ & $\mathrm{C}$ & $\begin{array}{l}\text { Enable designers to accurately model concepts of cyber-physical systems like sensors } \\
\text { and actuators }\end{array}$ & DS \\
\hline $\begin{array}{l}\text { (Jankovic et al., } \\
\text { 2015) }\end{array}$ & J & $\begin{array}{l}\text { Allow a formal modelling of informations used and generated in cross-organizational } \\
\text { BPs }\end{array}$ & DS \\
\hline $\begin{array}{l}\text { (Laue \& Mueller, } \\
\text { 2016) }\end{array}$ & $\mathrm{C}$ & Allow simulation of processes according to different scenarios & $\operatorname{Imp}$ \\
\hline $\begin{array}{l}\text { (Maines et al., } \\
\text { 2016) }\end{array}$ & $\mathrm{C}$ & Specify accurately all cyber security requirements across the third dimension & $\operatorname{Imp}$ \\
\hline $\begin{array}{l}\text { (Mandal et al., } \\
\text { 2017) }\end{array}$ & $\mathrm{C}$ & $\begin{array}{l}\text { Propose a model for event handling based on explicit subscriptions and event } \\
\text { buffering }\end{array}$ & $\operatorname{Imp}$ \\
\hline $\begin{array}{l}\text { (Martinho \& } \\
\text { Domingos, 2014) }\end{array}$ & J & Specify information quality and resource cost in loT-aware processes & DS \\
\hline $\begin{array}{l}\text { (Martinho et al., } \\
\text { 2015) }\end{array}$ & J & Ensure a controlled flexibility by defining where and how a BP can be changed & $\operatorname{Imp}$ \\
\hline $\begin{array}{l}\text { (Mazzola et al., } \\
\text { 2017) }\end{array}$ & $\mathrm{C}$ & $\begin{array}{l}\text { Employ an on-demand fault compensation mechanism through a Cloud-based } \\
\text { execution }\end{array}$ & $\operatorname{Imp}$ \\
\hline $\begin{array}{l}\text { (Merino et al., } \\
\text { 2016) }\end{array}$ & $\mathrm{C}$ & $\begin{array}{l}\text { Define probe-oriented features to convey more informations about activities in a } \\
\text { machine-understandable format }\end{array}$ & $\operatorname{Imp}$ \\
\hline $\begin{array}{l}\text { (Meyer et al., } \\
\text { 2015) }\end{array}$ & C & Integrate IoT technologies and concepts within BP models & DS \\
\hline $\begin{array}{l}\text { (Neumann et al., } \\
\text { 2016) }\end{array}$ & $\mathrm{C}$ & Model intraoperative surgical workflow in integrated operating rooms & DS \\
\hline
\end{tabular}




\begin{tabular}{|c|c|c|c|}
\hline $\begin{array}{l}\text { (Onggo et al., } \\
\text { 2017) }\end{array}$ & J & $\begin{array}{l}\text { Support the modelling of complex healthcare processes that require an explicit } \\
\text { representation of queues and data-driven decision points }\end{array}$ & DS \\
\hline $\begin{array}{l}\text { (Polderdijk et al., } \\
\text { 2018) }\end{array}$ & $\mathrm{C}$ & $\begin{array}{l}\text { Visualize and analyse human physical risks (e.g. heavy lifting) in manufacturing } \\
\text { processes }\end{array}$ & DS \\
\hline $\begin{array}{l}\text { (Pufahl \& Weske, } \\
\text { 2016) }\end{array}$ & $\mathrm{C}$ & $\begin{array}{l}\text { Integrate batch processing into BPMN by synchronizing the execution of a several } \\
\text { process instances }\end{array}$ & $\operatorname{Imp}$ \\
\hline $\begin{array}{l}\text { (Pullonen \& al., } \\
\text { 2017) }\end{array}$ & $\mathrm{C}$ & Secure processes by indicating the risks of private data leaks & Imp \\
\hline $\begin{array}{l}\text { (Ramos-Merino \& } \\
\text { al., 2018) }\end{array}$ & $\mathrm{J}$ & $\begin{array}{l}\text { Describe in a machine-understandable way a set of variables to be monitored during } \\
\text { BP execution }\end{array}$ & $\operatorname{Imp}$ \\
\hline $\begin{array}{l}\text { (Ramos-Merino \& } \\
\text { al., 2018) }\end{array}$ & J & Deal with the problem of ambiguities in representing hospital protocols & DS \\
\hline (Rekik \& al., 2016) & C & Specify BP requirements in order to outsource them to the best Cloud providers & $\operatorname{Imp}$ \\
\hline $\begin{array}{l}\text { (Respicio \& } \\
\text { Domingos, 2015) }\end{array}$ & J & Enrich BPMN with reliability informations to analyse alternatives at design time & $\operatorname{Imp}$ \\
\hline $\begin{array}{l}\text { (Salles et al., } \\
\text { 2018) }\end{array}$ & J & $\begin{array}{l}\text { Embody non-functional requirements and organizational goals through business level } \\
\text { agreements (BLAs) }\end{array}$ & $\operatorname{Imp}$ \\
\hline $\begin{array}{l}\text { (Salnitri et al., } \\
\text { 2017) }\end{array}$ & J & Express security aspects like integrity, accountability, and auditability & $\operatorname{Imp}$ \\
\hline $\begin{array}{l}\text { (Sang \& Zhou, } \\
\text { 2015) }\end{array}$ & $\mathrm{C}$ & Specify security requirements within healthcare processes & DS \\
\hline $\begin{array}{l}\text { (Tranquillini et al., } \\
\text { 2015) }\end{array}$ & J & $\begin{array}{l}\text { Program crowdsourcing in which multiple workers must coordinate to perform a } \\
\text { composite task }\end{array}$ & DS \\
\hline $\begin{array}{l}\text { (Vogel et al., } \\
\text { 2018) }\end{array}$ & $\mathrm{C}$ & Document and model smart glasses-based processes & DS \\
\hline $\begin{array}{l}\text { (Yahya et al., } \\
\text { 2015) }\end{array}$ & $\mathrm{C}$ & Integrate web 2.0 features and technologies in social BPs & DS \\
\hline $\begin{array}{l}\text { (Yahya et al., } \\
\text { 2018) }\end{array}$ & $\mathrm{Ch}$ & $\begin{array}{l}\text { Enrich BP models with social aspects by considering web } 2.0 \text { features (collaboration, } \\
\text { knowledge sharing, etc.) }\end{array}$ & DS \\
\hline $\begin{array}{l}\text { (Yousfi et al., } \\
2015 \text { ) }\end{array}$ & J & $\begin{array}{l}\text { Represent BPs that use technologies of ubiquitous computing like sensors and smart } \\
\text { readers }\end{array}$ & DS \\
\hline $\begin{array}{l}\text { (Yousfi et al., } \\
2016)\end{array}$ & J & Enable BPMN to represent accurately ubiquitous processes. & DS \\
\hline $\begin{array}{l}\text { (Zerbato etl al., } \\
2015)\end{array}$ & $\mathrm{C}$ & Add the time dimension in the modelling of healthcare processes related data & DS \\
\hline
\end{tabular}

Figures 4 and 5 represent the distribution of BPMN extensions by publication type and category, respectively.

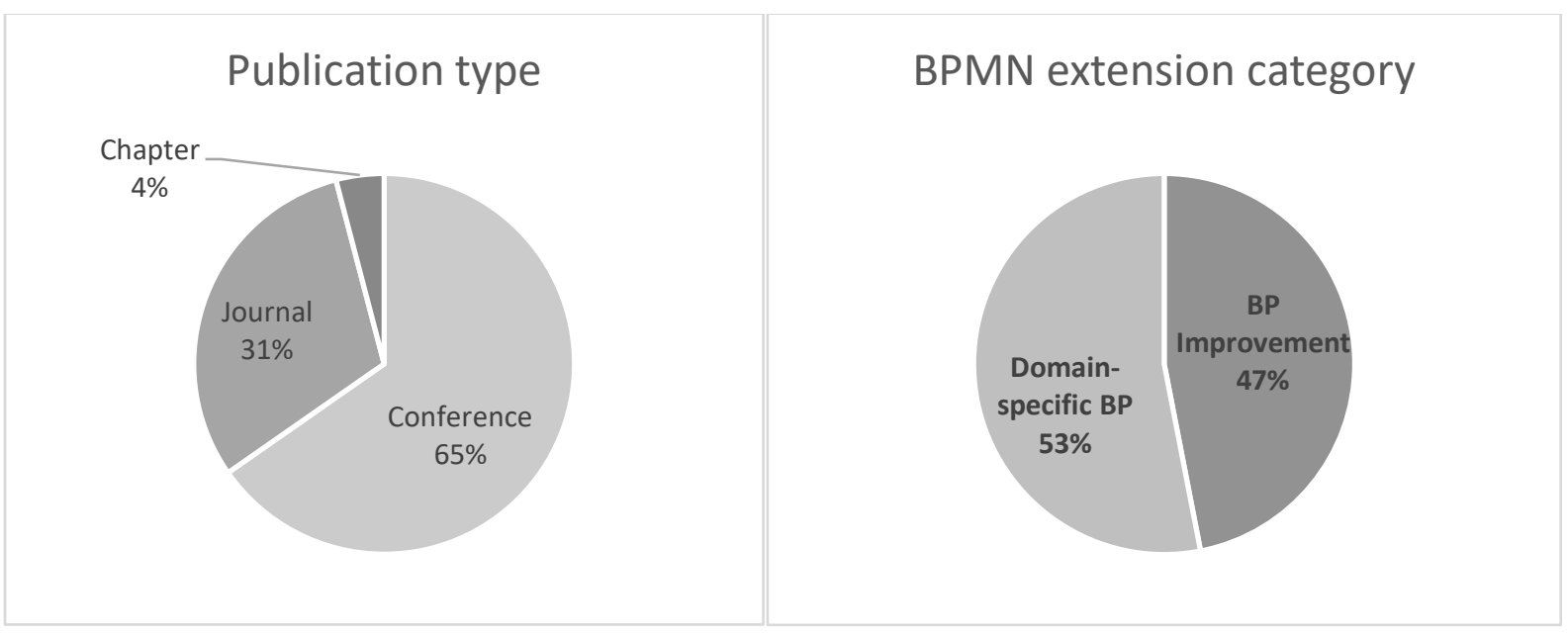

Figure 4. Distribution of BPMN extensions according to the type of publication
Figure 5. Distribution of BPMN extensions according to their category 
We notice that the majority of extensions are published in conferences. We can explain this by the fact that BPMN extensions are not consistent enough in terms of contribution to be submitted to journals. There is also the lack of maturity in this research area since BPMN is a relatively recent language (standardized by the OMG in 2006) compared to other languages like UML, which was standardized by the OMG in 1997. On the other hand, the distribution by category is balanced since some researchers are interested in the processes of a particular field while others aim to improve BPMN language or contribute to BPM activities.

In table 2, the BPMN extensions are compared according to their name, main domain, demonstration, implementation, and evaluation.

Table 2. Comparison of BPMN extensions according to their name, domain, demonstration, implementation, and evaluation

\begin{tabular}{|c|c|c|c|c|c|}
\hline $\begin{array}{l}\text { BPMN } \\
\text { extension }\end{array}$ & $\begin{array}{l}\text { Extension } \\
\text { name }\end{array}$ & Main domain & Illustrative example & Implementation & Evaluation \\
\hline $\begin{array}{l}\text { (Anseeuw \& al., } \\
\text { 2015) }\end{array}$ & / & Monitoring & Abstract example & No & No \\
\hline $\begin{array}{l}\text { (Arevalo et al., } \\
\text { 2016) }\end{array}$ & / & Time dimension & $\begin{array}{l}\text { Event management of an } \\
\text { organization }\end{array}$ & No & $\begin{array}{l}\text { Comparison } \\
\text { with other } \\
\text { approaches }\end{array}$ \\
\hline $\begin{array}{l}\text { (Ben Hassen et } \\
\text { al., 2017a) }\end{array}$ & BPMN4KM & $\begin{array}{l}\text { Knowledge } \\
\text { management }\end{array}$ & Care of disabled children & BPMN2 Modeler & No \\
\hline $\begin{array}{l}\text { (Ben said et al., } \\
\text { 2017) }\end{array}$ & BPMN4V & Flexibility & Radiological examination & BPMN2 Modeler & No \\
\hline $\begin{array}{l}\text { (Betke \& Seifert, } \\
\text { 2017) }\end{array}$ & / & $\begin{array}{l}\text { Disaster } \\
\text { response } \\
\text { management }\end{array}$ & No & No & No \\
\hline $\begin{array}{l}\text { (Bocciarelli et } \\
\text { al., 2017a) }\end{array}$ & / & $\begin{array}{l}\text { Cyber physical } \\
\text { systems }\end{array}$ & $3 \mathrm{D}$ printing & No & No \\
\hline $\begin{array}{l}\text { (Bocciarelli \& } \\
\text { al., 2017b) }\end{array}$ & / & Data exchange & $\begin{array}{l}\text { Production of hardware } \\
\text { components }\end{array}$ & No & No \\
\hline $\begin{array}{l}\text { (Bocciarelli et } \\
\text { al., 2016) }\end{array}$ & PyBPMN & $\mathrm{BP}$ resources & $\begin{array}{l}\text { Delivery of hard copy } \\
\text { contracts }\end{array}$ & No & No \\
\hline $\begin{array}{l}\text { (Braun \& } \\
\text { Esswein, 2015) }\end{array}$ & / & Complexity & Stroke diagnosis & No & No \\
\hline $\begin{array}{l}\text { (Braun et al., } \\
\text { 2015) }\end{array}$ & / & Healthcare & Wisdom tooth treatment & No & $\begin{array}{l}\text { Comparison } \\
\text { with a DSML }\end{array}$ \\
\hline $\begin{array}{l}\text { (Braun et al., } \\
\text { 2016) }\end{array}$ & BPMN4CP & Healthcare & Stroke care & No & no \\
\hline $\begin{array}{l}\text { (Cartelli et al., } \\
\text { 2016) }\end{array}$ & BPSIM & Simulation & $\begin{array}{l}\text { Releasing a construction } \\
\text { permit }\end{array}$ & New tool & no \\
\hline $\begin{array}{l}\text { (Carvalho et al., } \\
\text { 2017) }\end{array}$ & AO-BPM & Complexity & $\begin{array}{l}\text { University administrative } \\
\text { services }\end{array}$ & Draw.io & $\begin{array}{l}\text { Comparison } \\
\text { with BPMN }\end{array}$ \\
\hline $\begin{array}{l}\text { (Chergui \& } \\
\text { Benslimane, } \\
2018 \text { ) }\end{array}$ & / & Security & $\begin{array}{l}\text { Patient admission in a } \\
\text { hospital }\end{array}$ & No & $\begin{array}{l}\text { Experiments } \\
\text { with students }\end{array}$ \\
\hline $\begin{array}{l}\text { (Chiu \& Wang, } \\
\text { 2015) }\end{array}$ & / & IoT & Temperature control & No & No \\
\hline $\begin{array}{l}\text { (D'Ambrogio et } \\
\text { al., 2016) }\end{array}$ & PyBPMN & Performance & Abstract example & BPMN2 Modeler & No \\
\hline $\begin{array}{l}\text { (De Giacomo et } \\
\text { al., 2015) }\end{array}$ & BPMN-D & Variability & Purchase order process & No & no \\
\hline $\begin{array}{l}\text { (Domingos et } \\
\text { al., 2016) }\end{array}$ & relyBPMN & Healthcare & $\begin{array}{l}\text { Ambient assisted living } \\
\text { healthcare }\end{array}$ & No & $\begin{array}{l}\text { Reliability } \\
\text { calculation }\end{array}$ \\
\hline
\end{tabular}




\begin{tabular}{|c|c|c|c|c|c|}
\hline $\begin{array}{l}\text { (Dorndorfer \& } \\
\text { Seel, 2017) }\end{array}$ & $\begin{array}{l}\text { Context4BP } \\
\mathrm{MN}\end{array}$ & Mobile devices & $\begin{array}{l}\text { Inspection and control of } \\
\text { employees }\end{array}$ & No & no \\
\hline $\begin{array}{l}\text { (Dukaric \& Juric, } \\
\text { 2018) }\end{array}$ & / & $\begin{array}{l}\text { Cloud } \\
\text { computing }\end{array}$ & Abstract axample & New tool & $\begin{array}{l}\text { Measuring } \\
\text { complexity } \\
\text { using various } \\
\text { metrics }\end{array}$ \\
\hline $\begin{array}{l}\text { (Graja et al., } \\
\text { 2016) } \\
\text { implemented in } \\
\text { (Graja et al., } \\
\text { 2017) }\end{array}$ & BPMN4CPS & $\begin{array}{l}\text { Cyber-physical } \\
\text { systems }\end{array}$ & Ambulance drone system & BPMN2 Modeler & no \\
\hline $\begin{array}{l}\text { (Jankovic et al., } \\
\text { 2015) }\end{array}$ & / & $\mathrm{BP}$ resources & $\begin{array}{l}\text { Collaborative shipping } \\
\text { process }\end{array}$ & No & No \\
\hline $\begin{array}{l}\text { (Laue \& } \\
\text { Mueller, 2016) }\end{array}$ & BPSIM & Simulation & $\begin{array}{l}\text { Choice of the report type } \\
\text { required }\end{array}$ & No & No \\
\hline $\begin{array}{l}\text { (Maines et al., } \\
\text { 2016) }\end{array}$ & / & Security & Air traffic management & No & $\begin{array}{l}\text { Experiments } \\
\text { with students }\end{array}$ \\
\hline $\begin{array}{l}\text { (Mandal et al., } \\
\text { 2017) }\end{array}$ & / & Event handling & Shipping goods by truck & Camunda & no \\
\hline $\begin{array}{l}\text { (Martinho \& } \\
\text { Domingos, } \\
2014 \text { ) }\end{array}$ & / & IoT & No & No & No \\
\hline $\begin{array}{l}\text { (Martinho et al., } \\
\text { 2015) }\end{array}$ & CF4BPMN & Flexibility & $\begin{array}{l}\text { Elaboration phase of the } \\
\text { unified process (UP) }\end{array}$ & No & no \\
\hline $\begin{array}{l}\text { (Mazzola et al., } \\
\text { 2017) }\end{array}$ & FCE4BPMN & BP flexibility & Key-holder production & New tool & No \\
\hline $\begin{array}{l}\text { (Merino et al., } \\
\text { 2016) }\end{array}$ & / & BP mining & $\begin{array}{l}\text { Manufacturing chain of } \\
\text { metal pieces }\end{array}$ & No & $\begin{array}{l}\text { Comparison } \\
\text { with BPMN }\end{array}$ \\
\hline $\begin{array}{l}\text { (Meyer et al., } \\
\text { 2015) }\end{array}$ & / & IoT & $\begin{array}{l}\text { Temperature } \\
\text { measurement with an loT } \\
\text { device }\end{array}$ & No & No \\
\hline $\begin{array}{l}\text { (Neumann et } \\
\text { al., 2016) }\end{array}$ & BPMNSIX & Healthcare & No & No & No \\
\hline $\begin{array}{l}\text { (Onggo et al., } \\
\text { 2017) }\end{array}$ & BPMN4SIM & Healthcare & $\begin{array}{l}\text { Emergency care of elderly } \\
\text { patients }\end{array}$ & No & no \\
\hline $\begin{array}{l}\text { (Polderdijk et } \\
\text { al., 2018) }\end{array}$ & / & Manufacturing & $\begin{array}{l}\text { Loading profiles on racks } \\
\text { (Company of Thomas } \\
\text { Regout Int) }\end{array}$ & Microsoft Visio & $\begin{array}{l}\text { Interviews } \\
\text { with experts }\end{array}$ \\
\hline $\begin{array}{l}\text { (Pufahl \& } \\
\text { Weske, 2016) }\end{array}$ & / & $\begin{array}{l}\text { Batch } \\
\text { processing }\end{array}$ & $\begin{array}{l}\text { Shipping of customers' } \\
\text { orders }\end{array}$ & Camunda & No \\
\hline $\begin{array}{l}\text { (Pullonen \& al., } \\
\text { 2017) }\end{array}$ & PE-BPMN & Security & $\begin{array}{l}\text { RapidGather mobile } \\
\text { application }\end{array}$ & No & No \\
\hline $\begin{array}{l}\text { (Ramos-Merino } \\
\& \text { al., 2018) }\end{array}$ & / & Healthcare & $\begin{array}{l}\text { Management of hazardous } \\
\text { drugs }\end{array}$ & No & $\begin{array}{l}\text { Expreiments } \\
\text { with experts }\end{array}$ \\
\hline $\begin{array}{l}\text { (Ramos-Merino } \\
\& \text { al., 2018) }\end{array}$ & BPMN-E2 & Monitoring & Parenteral nutrition & Graphviz & $\begin{array}{l}\text { Comparison } \\
\text { with BPMN }\end{array}$ \\
\hline $\begin{array}{l}\text { (Rekik \& al., } \\
\text { 2016) }\end{array}$ & OutyBPMN & BP outsourcing & Abstract example & BPMN2 Modeler & no \\
\hline $\begin{array}{l}\text { Respicio \& } \\
\text { Domingos, } \\
\text { 2015) }\end{array}$ & / & Reliability & Paper reviewing & No & No \\
\hline $\begin{array}{l}\text { (Salles et al., } \\
\text { 2018) }\end{array}$ & BLA@BPMN & $\begin{array}{l}\text { Quality of } \\
\text { service }\end{array}$ & Credit application & Bizagi & $\begin{array}{l}\text { Survey with } \\
\text { experts }\end{array}$ \\
\hline $\begin{array}{l}\text { (Salnitri et al., } \\
\text { 2017) }\end{array}$ & $\begin{array}{l}\text { SecBPMN- } \\
\mathrm{ml}\end{array}$ & Security & Air traffic management & New tool & $\begin{array}{l}\text { Experiment } \\
\text { with experts }\end{array}$ \\
\hline $\begin{array}{l}\text { (Sang \& Zhou, } \\
\text { 2015) }\end{array}$ & / & Healthcare & $\begin{array}{l}\text { Remote healthcare } \\
\text { monitor system }\end{array}$ & Activiti & No \\
\hline $\begin{array}{l}\text { (Tranquillini et } \\
\text { al., 2015) }\end{array}$ & $\begin{array}{l}\text { BPMN4Cro } \\
\text { wd }\end{array}$ & Crowdsourcing & $\begin{array}{l}\text { Crowd-based mining of } \\
\text { mashup model patterns }\end{array}$ & Activiti & no \\
\hline $\begin{array}{l}\text { (Vogel et al., } \\
\text { 2018) }\end{array}$ & BPMN4SGA & IoT & $\begin{array}{l}\text { Movement of goods in a } \\
\text { distribution center }\end{array}$ & No & No \\
\hline
\end{tabular}




\begin{tabular}{|l|l|l|l|l|l|}
\hline $\begin{array}{l}\text { (Yahya et al., } \\
2015)\end{array}$ & $\begin{array}{l}\text { BPMN4Soci } \\
\text { al }\end{array}$ & Social aspects & Online course planning & BPMN2 Modeler & No \\
\hline $\begin{array}{l}\text { (Yahya et al., } \\
2018)\end{array}$ & $\begin{array}{l}\text { BPMN4Soci } \\
\text { al }\end{array}$ & Social aspects & $\begin{array}{l}\text { Maintenance of devices in } \\
\text { a commercial company }\end{array}$ & BPMN2 Modeler & no \\
\hline $\begin{array}{l}\text { (Yousfi et al., } \\
2015)\end{array}$ & uBPMN & $\begin{array}{l}\text { Ubiquitous } \\
\text { computing }\end{array}$ & $\begin{array}{l}\text { Time banking (request } \\
\text { fulfillment) }\end{array}$ & No & $\begin{array}{l}\text { Comparison } \\
\text { with BPMN }\end{array}$ \\
\hline $\begin{array}{l}\text { (Yousfi et al., } \\
2016)\end{array}$ & uBPMN & $\begin{array}{l}\text { Ubiquitous } \\
\text { computing }\end{array}$ & Order eyeglass frames & No & no \\
\hline $\begin{array}{l}\text { (Zerbato etl al., } \\
2015)\end{array}$ & $/$ & Healthcare & $\begin{array}{l}\text { Catheter-related } \\
\text { bloodstream infections }\end{array}$ & No & No \\
\hline
\end{tabular}

In figure 6, we calculate number of BPMN extensions for each domain. We note that the targeted areas are very diversified as we have identified 189 different domains knowing that some close domains have been grouped together. For instance, the CPS, IoT, and Ubiquitous computing domains have been grouped since they all involve the interconnection of physical entities via a network. Extensions that specify the BP requirements in terms of performance, reliability, QoS, etc. have been considered in non-functional properties.

We also note in figure 6 that healthcare is among the most targeted areas. The interest in healthcare is explained by the fact that it is very broad, very sensitive, and highly regulated. Indeed, processes in this area manipulate medical data that must be archived, remain confidential and always available to caregivers for decision-making. In addition, this area is highly regulated by extraterritorial laws (e.g., Health Insurance Portability and Accountability Act (HIPAA), Health Information Technology for Economic and Clinical Health (HITECH), International Health Regulations (IHR) (WHO, 2007)) and by standards (e.g., the norm ISO 18308: 2011 (ISO, 2017) for structuring electronic health record).

The domains involving the interconnection of physical entities are also very focused since they are very trendy these last years and they have many specific characteristics that should be considered such as energy consumption constraints, limited resources, data transfer, etc. Another domain also targeted by BPMN extensions is the flexibility and variability of processes allowing their adaptation to changes of the environment that becomes more dynamic and unpredictable with increasingly demanding customers.

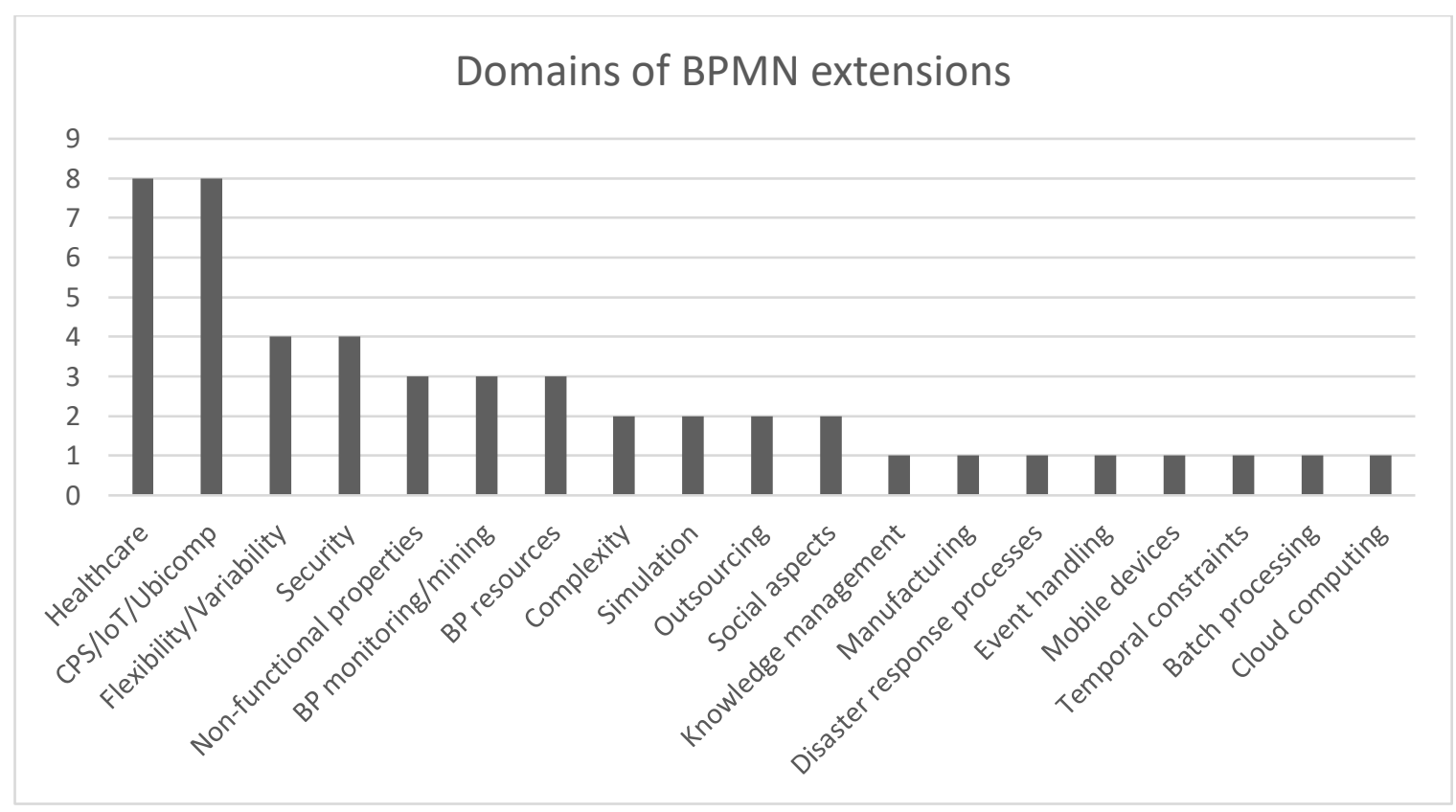

Figure 6. Distribution of BPMN extensions according to the targeted domain 
Figure 7 shows the tools used by authors to implement their extension. The BPMN2 Modeler tool is widely used. This is probably due to the ease and documentation offered by BPMN2 Modeler to help users in integrating the new elements of their extension. In fact, a tutorial on an extension example is explained in detail on the Eclipse website. To integrate new elements of an extension, it is enough to add a plugin to BPMN2 Modeler (which is itself a plugin integrated in Eclipse) contrary to other tools that require code injection or modification. Furthermore, many authors prefer to develop their own modelling tool although this method is costly and time-consuming.

In figure 8 , we observe that almost all the examples provided by the authors to demonstrate their BPMN extension are concrete processes (i.e., they are derived from real world scenarios).

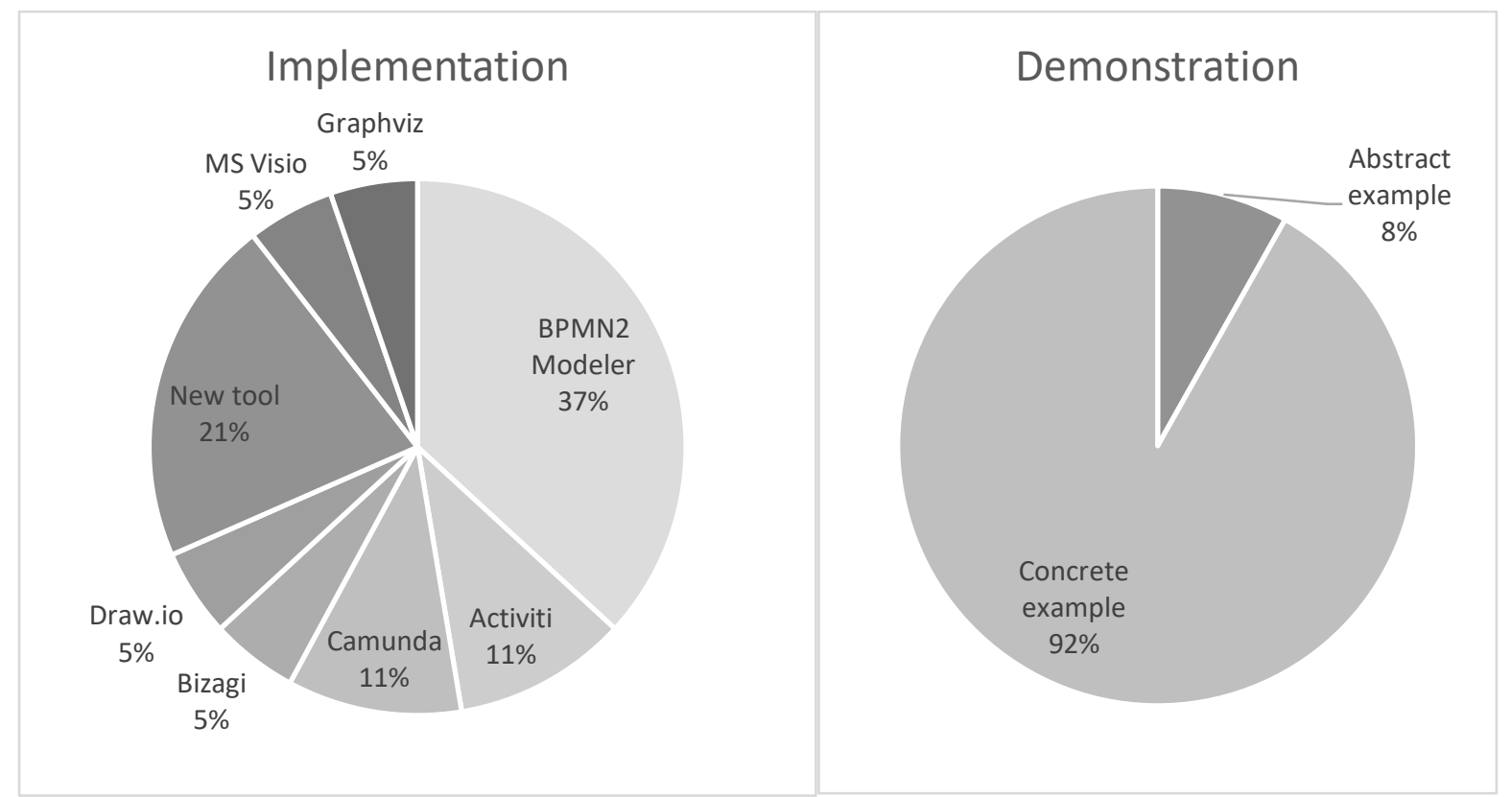

Figure 7. Distribution of BPMN extensions according to the implementation tool
Figure 8. Distribution of BPMN extensions according to the type of illustrative example

In table 3, we compare the BPMN extensions by taking into account their conformity, the formats adopted for their representation as well as the affected BPMN elements. For the conformity criterion, we specify between brackets whether one of the two methodologies of (Stroppi et al., 2011) or (Braun \& Schlieter, 2014) has been followed.

Table 3. Comparison of BPMN extensions according to their conformity, representation, and affected BPMN elements

\begin{tabular}{|c|c|c|c|}
\hline $\begin{array}{c}\text { BPMN } \\
\text { extension }\end{array}$ & Conformity & $\begin{array}{c}\text { Extension } \\
\text { representation }\end{array}$ & Affected elements \\
\hline
\end{tabular}




\begin{tabular}{|c|c|c|c|c|c|c|c|c|c|c|c|c|c|c|c|}
\hline & & 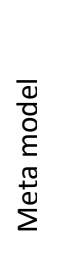 & 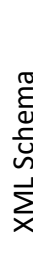 & 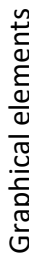 & 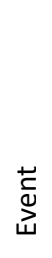 & $\sum_{\substack{Z\\
}}^{\geq}$ & 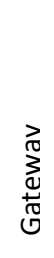 & 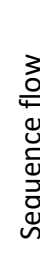 & 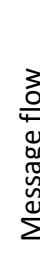 & 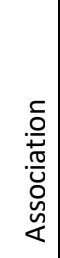 & $\overline{8}$ & 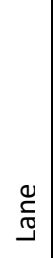 & $\begin{array}{l}\stackrel{U}{0} \\
\frac{\pi}{0} \\
0 \\
0 \\
\frac{\pi}{0} \\
0\end{array}$ & $\begin{array}{l}\text { 이 } \\
\text { 임 }\end{array}$ & 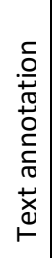 \\
\hline $\begin{array}{l}\text { (Anseeuw \& al., } \\
\text { 2015) }\end{array}$ & No & & & $x$ & $x$ & $x$ & $x$ & $x$ & & & & & $x$ & & \\
\hline $\begin{array}{l}\text { (Arevalo et al., } \\
\text { 2016) }\end{array}$ & No & $x$ & & $x$ & $x$ & $\mathrm{x}$ & $x$ & $x$ & & & & & & & \\
\hline $\begin{array}{l}\text { (Ben Hassen et } \\
\text { al., 2017a) }\end{array}$ & Yes (Stroppi) & $x$ & $x$ & $X$ & & $X$ & & & $x$ & & & & & & \\
\hline $\begin{array}{l}\text { (Ben said et al., } \\
\text { 2017) }\end{array}$ & Yes & $x$ & & $x$ & $x$ & $X$ & & & & & $x$ & & & & \\
\hline $\begin{array}{l}\text { (Betke \& Seifert, } \\
\text { 2017) }\end{array}$ & Yes (Stroppi) & $x$ & & $x$ & & $\mathrm{x}$ & & & & & & & & & \\
\hline $\begin{array}{l}\text { (Bocciarelli et } \\
\text { al., 2017a) }\end{array}$ & No & $x$ & & & & $\mathrm{x}$ & & & & & & & & & $X$ \\
\hline $\begin{array}{l}\text { (Bocciarelli \& } \\
\text { al., 2017b) }\end{array}$ & No & $x$ & & & & & & & $x$ & & & & & & \\
\hline $\begin{array}{l}\text { (Bocciarelli et } \\
\text { al., 2016) }\end{array}$ & No & $x$ & & & & $\mathrm{x}$ & & & & & & & & & $x$ \\
\hline $\begin{array}{l}\text { (Braun \& } \\
\text { Esswein, 2015) }\end{array}$ & Yes (Stroppi) & $x$ & & $x$ & & $x$ & & & & & & & $x$ & & \\
\hline $\begin{array}{l}\text { (Braun et al., } \\
\text { 2015) }\end{array}$ & Yes (Braun) & $x$ & & $x$ & & $\mathrm{x}$ & $x$ & & & & & & $x$ & & \\
\hline $\begin{array}{l}\text { (Braun et al., } \\
2016 \text { ) }\end{array}$ & Yes (Braun) & $x$ & & $X$ & & $x$ & $x$ & & & $X$ & & & $X$ & & \\
\hline $\begin{array}{l}\text { (Cartelli et al., } \\
\text { 2016) }\end{array}$ & Yes & & $x$ & & $x$ & $x$ & $x$ & $x$ & & & & $x$ & & & \\
\hline $\begin{array}{l}\text { (Carvalho et al., } \\
\text { 2018) }\end{array}$ & No & & & $x$ & & $x$ & & $x$ & & $x$ & $x$ & $x$ & & & \\
\hline $\begin{array}{l}\text { (Chergui \& } \\
\text { Benslimane, } \\
2018 \text { ) }\end{array}$ & Yes (Stroppi) & $x$ & $X$ & $X$ & & $X$ & & & & & & & & & \\
\hline $\begin{array}{l}\text { (Chiu \& Wang, } \\
\text { 2015) }\end{array}$ & No & $x$ & & $x$ & $x$ & & & & & & & & & & \\
\hline $\begin{array}{l}\text { (D'Ambrogio et } \\
\text { al., 2016) }\end{array}$ & No & $x$ & & & $x$ & & & & & & & & $x$ & & \\
\hline $\begin{array}{l}\text { (De Giacomo et } \\
\text { al., 2015) }\end{array}$ & No & & & $x$ & & $\mathrm{x}$ & & $x$ & & & & & & & \\
\hline $\begin{array}{l}\text { (Domingos et } \\
\text { al., 2016) }\end{array}$ & Yes (Stroppi) & $x$ & $x$ & & $x$ & $x$ & $x$ & $x$ & & & & & & & \\
\hline $\begin{array}{l}\text { (Dorndorfer \& } \\
\text { Seel, 2017) }\end{array}$ & Yes & $x$ & & $x$ & $x$ & $x$ & & & & & & & & $x$ & $X$ \\
\hline $\begin{array}{l}\text { (Dukaric \& Juric, } \\
\text { 2018) }\end{array}$ & Yes & $x$ & $x$ & $x$ & & $\mathrm{x}$ & & & & & & & & & \\
\hline $\begin{array}{l}\text { (Graja et al., } \\
\text { 2016) } \\
\text { implemented in } \\
\text { (Graja et al., } \\
\text { 2017) }\end{array}$ & Yes & $x$ & & $x$ & $x$ & $\mathrm{X}$ & & & & & $x$ & $x$ & & & \\
\hline
\end{tabular}




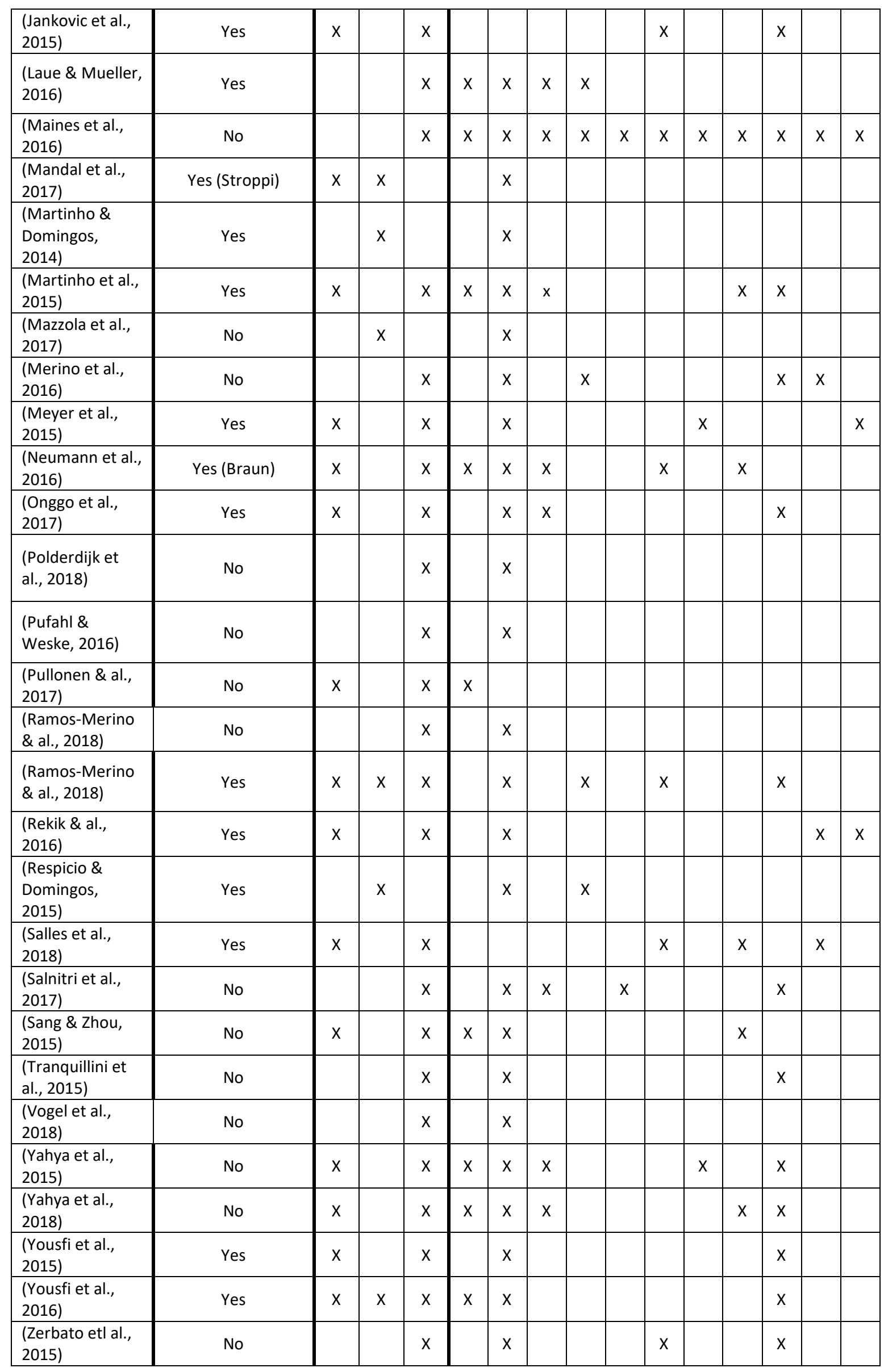


We notice in figure 9 that most authors represent their BPMN extension in the form of a meta-model and graphical elements. However, the XML Schema is little used despite it specifies an interchange and storage format for BP models (OMG, 2013).

As for the affected BPMN elements, we see in figure 10 that the Activity element is by far the most affected by BPMN extensions. In fact, it is affected in almost all extensions (43 out of 49). This heavy use is probably due to the fact that the Activity element defines the works performed within BPs whether atomic (task) or compound (sub-process) (OMG, 2013). We also perceive that Event and Data Object elements are widely used. Indeed, the first element allows to take into account the external environment by defining everything that can occur during the BP execution and that may have an impact on the process flow while the second element provides information about what activities require to be performed and what they produce (OMG, 2013).

More generally, we observe that the Flow Objects category, which includes Event, Activity, and Gateway elements, is the most affected with an average of 25 uses among 49 BPMN extensions. We find this result logical as this category encompasses the main elements for defining process behaviour (OMG, 2013).

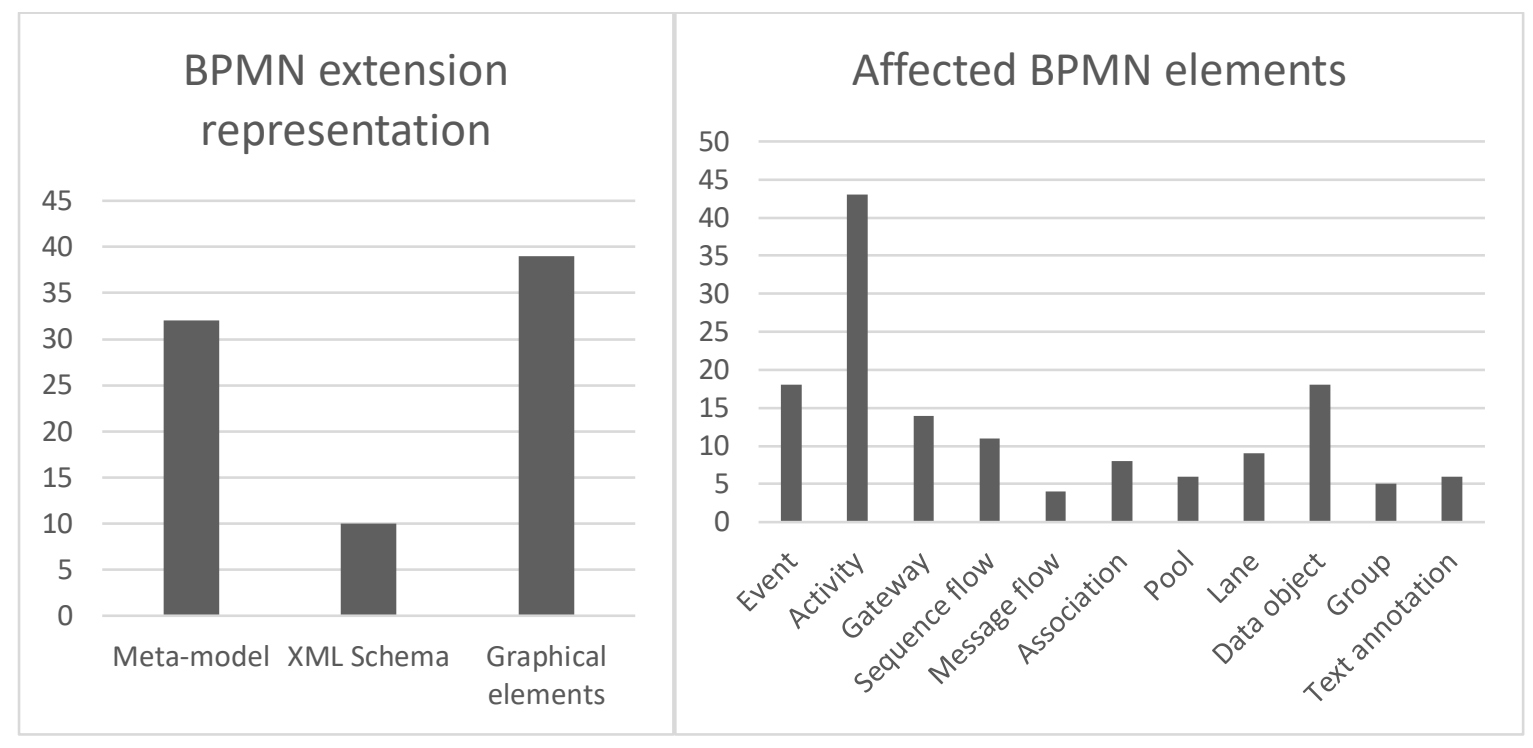

Figure 9. The number of uses of each format for representing BPMN extensions

Figure 10. The number of affectations of BPMN elements by extensions

In figure 11, we based on tables 2 and 3 to evaluate BPMN extensions in terms of conformity, demonstration, evaluation, and implementation. The statistics reveal that:

- Less than half of the extensions (47\%) comply with the BPMN extension mechanism what hampers comprehensibility and comparability of BPMN extensions and impedes their straightforward integration in modelling tools. This low conformance rate is due to the absence of a standard methodology for developing extensions since the OMG specifies only the representation formats, namely the MOF meta-model and the XML Schema. Given also the number of meta-models defined in the BPMN documentation, authors have trouble finding which one to extend. Moreover, authors have to know the basics of XML Schema to be able to use it in the representation of their extension. Other reasons are also possible such as the length of BPMN documentation (more than 500 pages) and the lack of clarity on how to extend the meta-models. 
- Apart from three BPMN extensions, all the others have been demonstrated through examples that are in most cases concrete. This helps to understand the usefulness of the proposed extensions.

- Only $29 \%$ of BPMN extensions were evaluated. Generally, authors evaluate their extension by comparing it with the BPMN language, with other extensions, or with a domain-specific modelling language (DSML). For instance, (Carvalho et al., 2018) compared their extension with BPMN through LOC metrics, (Braun et al., 2015) compared their extension with a DSML while the extension proposed in (Arevalo et al., 2016) was compared with another BPMN extension. The comparison is often used because it allows to highlight the added values of the proposed extension. Other authors opt for interviews and questionnaires with a group of experts. For example (Salles et al., 2018) used the Goal Question Metric (GQM) technique while (Salnitri et al., 2017) opted for an online survey left for 20 days.

- Only $39 \%$ of extensions have been implemented despite the fact that most modelling tools such as Activiti, Bizagi, and BPMN2 Modeler are extensible and offer the possibility of adding new graphic elements and integrating them into their palette. We have also noted that several authors mention at the end of the paper that they plan to implement their extension in the future. Therefore, we can expect publications implementing the extensions already proposed as the authors did in (Graja et al., 2016) and (Graja et al., 2017).

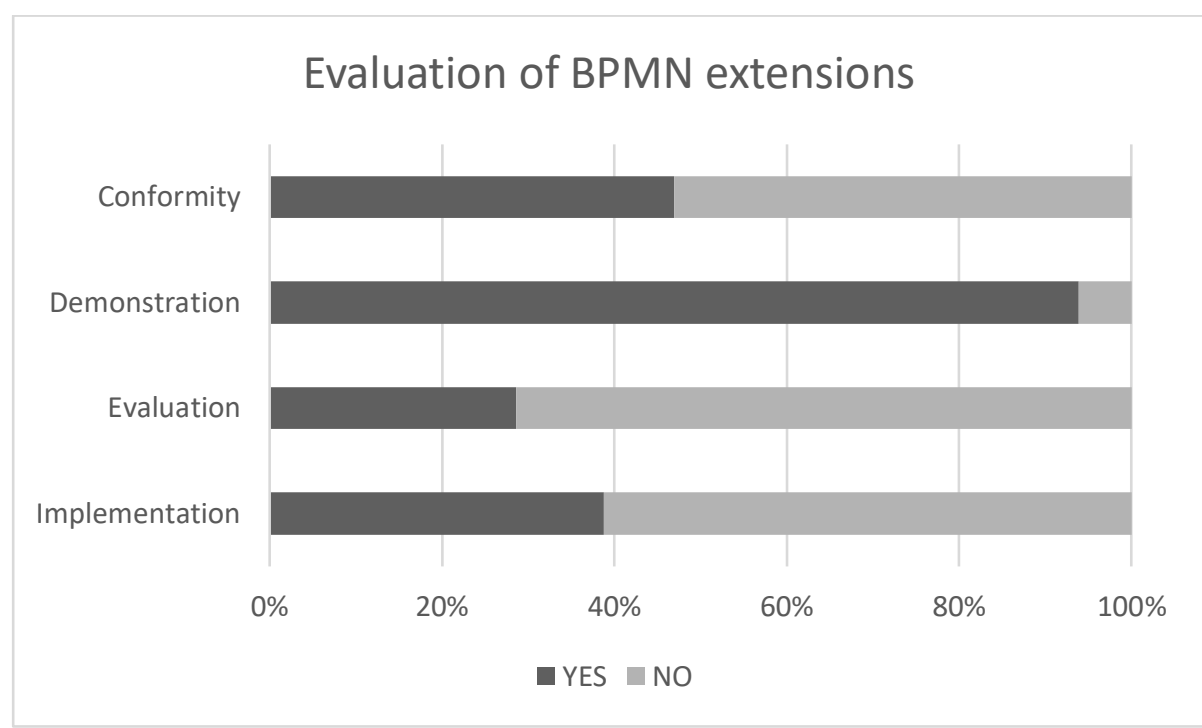

Figure 11. Evaluation of BPMN extensions according to conformity, demonstration, evaluation, and implementation criteria

In addition, we have noticed that all authors explain the need to extend the BPMN language in their context and justify each of the new added elements.

\section{Related work}

To the best of the authors' knowledge, (Braun \& Esswein, 2014) is the only work that has conducted a literature review on BPMN extensions through a descriptive analysis and classification of 30 domainspecific BPMN extensions. We are very grateful to the authors for their efforts. However, we found the following limitations:

- The classification of 11 extensions published before the existence of the extension mechanism that was introduced in the version BPMN 2.0 in January 2011. Therefore, these extensions cannot be considered as non-conform. 
- The authors focused on extensions devoted for specific domains and neglected those that aim to improve the BPMN language or contribute to BPM activities.

- The non-consideration of the XML Schema format in representing BPMN extensions.

- The non-consideration of certain criteria such as the implementation, demonstration, and evaluation of BPMN extensions.

- The extended BPMN elements that were compared are not of the same level. For example, the Task element was compared with the Activity element, yet Task is a subtype of Activity. Artefact was also compared with Text annotation, which is an element of the Artefact category. To obtain significant results, it is required to compare either BPMN elements or their categories.

- The literature review is not exhaustive as some publications such as (Rodriguez et al., 2012; Zor et al., 2011 ; Baumegrass et al., 2014) have not been examined despite they propose a BPMN extension and are published over the covered timespan (2007 to 2014).

The objective of our literature review is to complement that of (Braun \& Esswein, 2014) in terms of both literature and criteria. Indeed, the last paper treated in the literature review of (Braun \& Esswein, 2014) was published on November 2, 2014. Therefore, in our literature review, we examined all BPMN extensions published after this date.

In order to see the evolution of extensions since the appearance of BPMN, we compared in figure 12 some of our results with those obtained by (Braun \& Esswein, 2014).

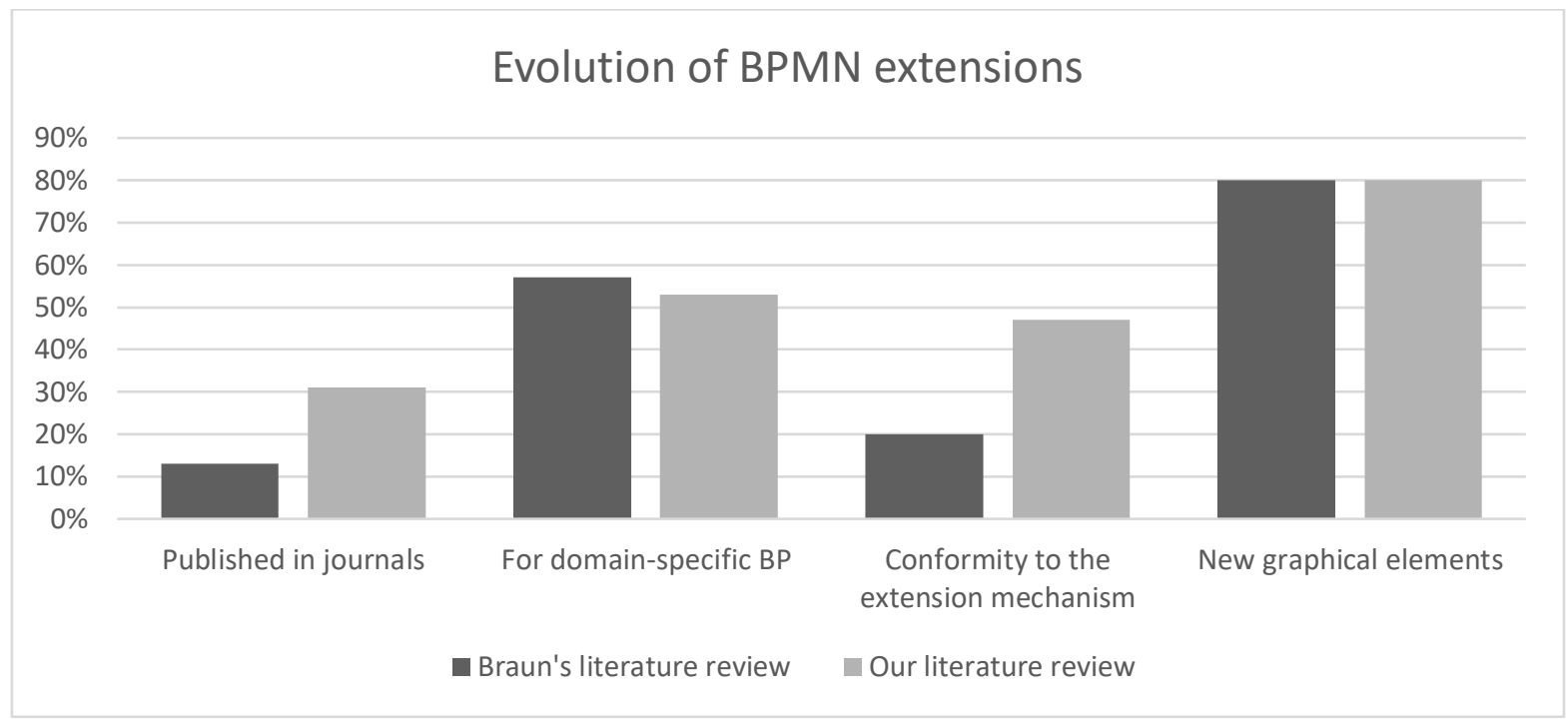

Figure 12. Evolution of BPMN extension characteristics over the past few years

From this comparison, we have noted the following points:

- The publication of BPMN extensions in journals has slightly increased. This proves that this research axis has gained in maturity and the extensions are more consistent, especially with their implementation and evaluation. However, this percentage remains low (31\%) and we believe that it will increase further in the coming years.

- The extensions reviewed in (Braun \& Esswein, 2014) are slightly more oriented towards specific domains than the improvement of BPMN. This is related to the choice of the literature of (Braun \& Esswein, 2014) which as we mentionned previously, focused on extensions dealing with processes of specific domains.

- Extensions are becoming more conform to the BPMN extension mechanism. This result is logical given that many extensions analyzed in (Braun \& Esswein) were published before the OMG introduced the extension mechanism and are considered non-conform. 
- New graphic elements are introduced in the majority of BPMN extensions, whether old (extensions treated in (Braun \& Esswein, 2014)) or recent (extensions treated in our SLR).

Furthermore, we noticed that the domains highly targeted by the extensions have changed. Indeed, in (Braun \& Esswein, 2014), the two most targeted fields are performance and risk management, whereas in our SLR, healthcare and interconnection of physical entities are the most targeted.

There are also work that have conducted a literature review on the extension of other languages. For instance, (Kopp et al., 2011) have classified 62 BPEL extensions while (Pardillo, 2010) has reviewed UML-profiling practices based on the analysis of 39 publications.

Some researchers have conducted literature reviews focusing on business process modelling more generally and independently of a specific language. For example, (Aguilar Saven, 2004) reviewed the main process modelling techniques existing in the literature and classified them according to their purpose. An SLR was performed in (Moreno-Montes de Oca et al., 2015) for determining what quality aspects have been addressed in BP modelling and which gaps remain to be covered.

(Aldin \& Cesare, 2011) have surveyed the existing literature that addresses the problem of reusability in BP modelling for discovering reusable artefacts in the form of patterns. In the same field, (Zaaboub Haddar et al., 2014) have given an insight into existing types of reusable artefacts, their limitations, and reuse context. This latter two works intend to assist BP designers in choosing the appropriate types of reusable artefacts in their modelling project. Finally, a thorough SLR was reported in (Awadid \& Nurcan, 2017) on consistency requirements in BP modelling with a categorization of existing consistency approaches.

\section{Conclusion and future work}

BP modelling is arguably one of the major research areas in recent years as it takes an important part in the development of modern information systems. Although there exist several languages, BPMN has become the leader in BP modelling thanks to its expressiveness and simplicity. However, the latter provides generic elements and often needs to be extended. Since the development of a DSML from scratch is very time-consuming, a large number of extensions are proposed in the literature whether for dealing with domain-specific BPs (e.g., manufacturing, ubiquitous computing, healthcare) or for enhancing the BPMN language in terms of flexibility, security, complexity, etc.

In this paper, an SLR was conducted based on the guidelines depicted in (Kitchenham, 2007) in order to determine the current state of the art of BPMN extensions and identify their gaps. The extraction and filtering of papers resulted in a set of 49 BPMN extensions that were thoroughly examined and compared. Given that there already exists a literature review on BPMN extensions presented in (Braun \& Esswein, 2014), our SLR has complemented it by considering all extensions published after the last extension treated in (Braun \& Esswein, 2014 ). More precisely, we considered all BPMN extensions published between November 2014 and December 2018 in conference proceedings, journals, and chapters.

The BPMN extensions were evaluated and compared according to a set of criteria, including among others, the objective, targeted domain, representation formats, affected BPMN elements, conformity to the extension mechanism, demonstration, implementation, evaluation, etc. Furthermore, our SLR can serve as an inventory for BP designers by helping them in choosing the BPMN extension that best suits their needs and avoid developing a new extension. After the presentation, interpretation, and analysis of the obtained results, we can deduce essentially the following points:

- The targeted areas and objectives are very diversified.

- Despite a slight improvement in recent years, less than half of the extensions are conform to the BPMN extension mechanism. 
- The XML Schema is often overlooked when representing BPMN extensions, yet it specifies an interchange format for BP models.

- The majority of BPMN extensions are graphically represented and demonstrated through concrete examples, which allows understanding their usefulness.

- BPMN extensions are rarely evaluated and little implemented despite the existence of several extensible modelling tools.

Based on these deductions, we suggest the following recommendations that may bridge the identified gaps and advance the field of BPMN extensions:

- Authors must strictly adhere to the BPMN extension mechanism so as to provide a valid extension and enable BP model exchangeability.

- In addition to the MOF meta-model and XML schema formats, a clear methodology should be provided to guide authors throughout the development of their extension from the specification of target domain requirements to the implementation.

- It is desirable to define standard metrics for the evaluation and comparison of the proposed BPMN extensions.

- Finally, authors should make more effort in the implementation of their extension by integrating it into at least one modelling tool in order to prove its feasibility.

For future work, we plan to strengthen this literature review by taking into account other evaluation criteria and by covering more literature such as extensions not written in English and those proposed in master and doctoral theses. We also envisage to conduct a literature review on existing DSMLs and the extension of other BP-related languages like BPEL and UML. Finally, another literature review on BPMN extensions should be performed in the coming years in order to see the evolution of BPMN extension features.

\section{References}

Aguilar Saven, R.S. 'Business process modelling: review and framework', Int. J. Prod. Econ. 90 (2004) 129-149.

Aldin, L., \& Cesare, S. (2011). 'A literature review on business process modelling: new frontiers of reusability', Enterprise Information Systems, 5:3, 359-383

Anseeuw, J., Van Seghbroeck, G., Volckaert, B., \& De Turck, F. (2015). BPMN extensions for decentralized execution and monitoring of business processes. Proceedings of the 5th International Conference on Cloud Computing and Services Science (CLOSER 2015), (pp. 304-309)

Arevalo, C., Escalona, M.J., Ramos, I., \& Domínguez-Munoz, M., 'A metamodel to integrate business processes time perspective in BPMN 2. 0', Information and Software Technology 77 (2016) 17-33

Awadid, A. \& Nurcan, S. 'Consistency requirements in business process modeling: a thorough overview'. Softw Syst Model (2017)

Baumgrass, A., Herzberg, N., Meyer, A. and Weske, M. (2014) 'BPMN Extension for Business Process Monitoring' in Proceedings of Enterprise Modelling and Information Systems Architectures (EMISA), Luxembourg, pp.85-95.

Ben Hassen M., Keskes M., Turki M., Gargouri F., (2017a), 'BPMN4KM: Design and Implementation of a BPMN Extension for Modeling the Knowledge Perspective of Sensitive Business Processes', Procedia Computer Science, Volume 121, Pages 1119-1134

Ben Hassen M., Turki M., Gargouri F. (2017b), 'A BPMN Extension for Integrating Knowledge Dimension in Sensitive Business Process Models', European, Mediterranean, and Middle Eastern Conference on Information Systems (EMCIS 2017), pp 559-578 
Ben Hassen M., Turki M., Gargouri F. (2017c). 'Using Core Ontologies for Extending Sensitive Business Process Modeling with the Knowledge Perspective', Proceedings of the Fifth European Conference on the Engineering of Computer-Based Systems (ECBS'17), Article 2, August 2017

Ben Said I., Chaâbane M.A., Andonoff E., Bouaziz R. (2017). 'BPMN4V for Modeling and Handling Versions of BPMN Collaborations and Choreographies', International Conference on E-Business and Telecommunications (ICETE), 2016, pp 99-123

Betke, H. \& Seifert, M, 2017, 'BPMN for Disaster Response Processes - A methodical extension', Conference: INFORMATIK 2017, September 2017, 1311-1324.

Bocciarelli, P., D'Ambrogio, A., Giglio, A., \& Paglia, E. (2017a). 'A BPMN extension for modeling Cyber-PhysicalProduction-Systems in the context of Industry 4.0'. 2017 IEEE 14th International Conference on Networking, Sensing and Control (ICNSC), 599-604. , May 2017, Calabria, Italy

Bocciarelli, P., D'Ambrogio, A., Giglio, A., \& Paglia, E., 'A BPMN Extension to Enable the Explicit Modeling of Task Resources', Proc. of the 2nd INCOSE Italia Conference on Systems Engineering (CIISE), pp. 40-47, 2016.

Bocciarelli, P., D'Ambrogio, A., Paglia, E., \& Giglio, A. 'An HLA-based BPMN extension for the specification of business process collaborations', Proceedings of the 21st International Symposium on Distributed Simulation and Real Time Applications (DS-RT '17), Pages 67-74, Rome, Italy, 2017b

Bocciarelli, P., D'Ambrogio, E., Giglio, A., \& A., Paglia. 'Towards performance-oriented perfective evolution of BPMN models', In Proceedings of the Symposium on Theory of Modeling and Simulation (TMS-DEVS), part of the SCS SpringSim 2016 conference, April 2016, Pasadena, CA, USA

Braun R., Esswein W. (2015), 'Towards Multi-Perspective Modeling with BPMN', Enterprise Engineering Working Conference (EEWC 2015), pp 67-81

Braun, R. and Esswein, W. (2014) 'Classification of Domain-Specific BPMN Extensions' in Proceedings of the 7th IFIP Working Conference on The Practice of Enterprise Modeling (PoEM), Manchester, UK, pp.42-57.

Braun, R., \& Schlieter, H. "Requirements-based development of bpmn extensions -the case of clinical pathways", 1st International Workshop on the Interrelations between Requirements Engineering and Business Process Management, 2014.

Braun, R., Schlieter, H., Burwitz, M., \& Esswein, W. (2016), 'BPMN4CP Revised-Extending BPMN for Multiperspective Modeling of Clinical Pathways', 49th Hawaii International Conference on System Sciences (HICSS), pp. 3249-3258, January 2016, Koloa, HI, USA

Braun, R., Schlieter, H., Burwitz, M., \& Esswein. W., (2015). 'Extending a Business Process Modeling Language for Domain-Specific Adaptation in Healthcare'. Wirtschaftsinformatik Proceedings 2015, pp. 468-481

Brucker, A. (2013). Integrating Security Aspects into Business Process Models. it - Information Technology it Information Technology, 55(6), pp. 239-246.

Cartelli V., Di Modica G., Tomarchio O. (2016), 'Extending the BPMN Specification to Support Cost-Centric Simulations of Business Processes', 7th International Joint Conference on Knowledge Discovery, Knowledge Engineering, and Knowledge Management IC3K 2015, pp 492-514, Lisbon, Portugal, November 2015

Carvalho L.P., Cappelli C., Santoro F.M. (2018) AO-BPM 2.0: Aspect Oriented Business Process Modeling. In: Teniente E., Weidlich M. (eds) Business Process Management Workshops. BPM 2017.

Chergui, M.E.A., \& Benslimane, S.M., (2018). “A Valid BPMN Extension for Supporting Security Requirements Based on Cyber Security Ontology". International Conference on Model and Data Engineering (MEDI), Springer, pp 219-232, October, Marrakesh, Morocco

Chiu, HH \& Wang, MS, 2015, 'Extending Event Elements of Business Process Model for Internet of Things', IEEE International Conference on Computer and Information Technology; Ubiquitous Computing and 
Communications; Dependable, Autonomic and Secure Computing; Pervasive Intelligence and Computing, October 2015, Liverpool, UK, 783-788

De Giacomo G., Dumas M., Maggi F.M., Montali M. (2015) ‘Declarative Process Modeling in BPMN'. International Conference on Advanced Information Systems Engineering (CAiSE 2015): Advanced Information Systems Engineering, pp 84-100

Domingos, D., Respicio A., \& Martinho R. (2016). 'Reliability of loT-Aware BPMN Healthcare Processes', In book: Internet of Things and Advanced Application in Healthcare, chapter 8, pp. 214-244, IGI Global

Dorndorfer, J., Seel, C. (2017): A Meta Model Based Extension of BPMN 2.0 for Mobile Context Sensitive Business Processes and Applications, in Leimeister, J.M.; Brenner, W. (Hrsg.): Proceedings der 13. Internationalen Tagung Wirtschaftsinformatik (WI 2017), St. Gallen, S. 301-315, February, St. Gallen, Switzerland

Dukaric, R., \& Juric, M.B. (2018). "BPMN extensions for automating cloud environments using a two-layer orchestration approach". Journal of Visual Languages and Computing, 47, 31-43

Graja, I., Kallel, S., Guermouche, N., \& Kacem, A.H. 'Bpmn4cps: A bpmn extension for modeling cyber-physical systems', Proceedings of 25th IEEE International Conference on Enabling Technologies: Infrastructures for Collaborative Enterprises WETICE, pp. 152-157, June 2016, Paris, France

Graja, I., Mechim, A., Kallel, S., Guermouchey, N., \& Kacem, A.H. 'Demonstrating BPMN4CPS: modeling and verification of cyber-physical systems', In Proceedings of the 14th IEEE Annual Consumer Communications \& Networking Conference (CCNC), January 2017, Las Vegas, NV, USA

Jankovic, M., Ljubicic, M., Anicic, N. and Marjanovic, Z., 'Enhancing BPMN 2.0 informational perspective to support interoperability for cross-organizational business processes'. Computer Science and Information Systems, 2015, 12(3), pp.1101-1120.

Kitchenham, B. (2007). 'Guidelines for performing Systematic Literature Reviews in Software Engineering', version 2.3, technical report EBSE-2007-01, pp. 1-65, Keele University and Durham University.

Kopp, O., Gorlach, K., Karastoyanova, D., Leymann, F., Reiter, M., Schumm, D., Sonntag, M., Strauch, S., Unger, T., Wieland, M., \& Khalaf, R.: A classification of BPEL extensions. Journal of Systems Integration 2(4) (2011) 3\{28\}

Laue, R., \& Mueller, C. (2016). The Business Process Simulation Standard (BPSIM): Chances And Limits. In 30th European Conference on Modelling and Simulation ECMS, May 2016, Regensburg, Germany, pp. 413-418

Maines, C.L., Zhou, B., Tang, S., \& Shi, Q. "Adding a Third Dimension to BPMN as a means of Representing Cyber Security Requirements", 2016, 9th International Conference on Developments of E-Systems Engineering (DeSE), September 2016, Liverpool, UK

Mandal S., Weidlich M., Weske M. (2017), 'Events in Business Process Implementation: Early Subscription and Event Buffering', International Conference on Business Process Management (BPM 2017), pp 141-159, Barcelona

Martinho, R., Domingos, D., Varajao, J., 'CF4BPMN: a BPMN extension for controlled flexibility in business processes', Procedia Computer Science, Volume 64, 2015, Pages 1232-1239

Martinho, R., \& Domingos, D. 'Quality of Information and Access Cost of loT Resources in BPMN Processes', Procedia Technology, Volume 16, 2014, Pages 737-744

Mazzola, L., Kapahnke, P., Waibel, P., Hochreiner, C., \& Klusch, M. (2017). 'FCE4BPMN: On-demand QoS-based optimised process model execution in the cloud'. 2017 International Conference on Engineering, Technology and Innovation (ICE/ITMC), 305-314. June 2017, Funchal, Portugal

Merino M.R., Alvarez Sabucedo L.M., Santos Gago J.M., Alonso Rorís V.M. (2016), 'Extending BPMN Model for Improving Expressiveness and Machine-Understandability'. WorldCIST'16 - 4th World Conference on Information Systems and Technologies, In New Advances in Information Systems and Technologies, pp 297-306, March 2016, Recife, PE, Brazil 
Meyer S., Ruppen A., Hilty L. (2015), 'The Things of the Internet of Things in BPMN', International Conference on Advanced Information Systems Engineering (CAiSE 2015), pp 285-297

Moreno-Montes de Oca, I., Snoeck, M., Reijers, H. A., \& Rodríguez-Morffi, A. (2015). 'A systematic literature review of studies on business process modeling quality. Information and Software Technology', 58, 187-205.

Neumann, J., Rockstroh, M., Franke, S., \& Neumuth, T. 'BPMN SIX - a BPMN 2.0 surgical intervention extension: concept and design of a BPMN extension for intraoperative workflow modeling and execution in the integrated operating room'. In: 7th Workshop on Modeling and Monitoring of Computer Assisted Interventions (M2CAI), Athens, Greece; 2016

Object Management Group (OMG) (2013) 'Business Process Model and Notation (BPMN) Version 2.0.2' [online] http://www.omg.org/spec/BPMN/2.0.2/

Onggo, B. S. S., Proudlove, N.C., D’Ambrogio, A., Calabrese, A., Bisogno, .S., \& Levialdi Ghiron, N., 'A BPMN extension to support discrete-event simulation for healthcare applications: an explicit representation of queues, attributes and data-driven decision points', Journal of the Operational Research Society 69 (5), pp. 788-802, 2017

Pardillo, J. 'A systematic review on the definition of uml profiles'. In: Model Driven Engineering Languages and Systems. Springer (2010) 407\{422\}

Pillat, R.M., Oliviera, T.C., Alencar, P.S.C. and Cowan, D.D. (2015) 'BPMNt: A BPMN extension for specifying software process tailoring', Information and Software Technology, Vol. 57, pp.95-115.

Polderdijk, M., Vanderfeesten, I., Erasmus, J., Traganos, K., Bosch, T., Rhijn, G., \& Fahland, D. (2018) A Visualization of Human Physical Risks in Manufacturing Processes Using BPMN. In Business Process Management Workshops. BPM 2017.

Pufahl, L., \& Weske, M. (2016). 'Enabling Batch Processing in BPMN Processes'. BPM. In BPM Demos, pages 2833. Springer, 2016.

Pullonen, P., Matulevicius, R., \& Bogdanov, D. (2017). ‘PE-BPMN: Privacy-Enhanced Business Process Model and Notation', International Conference on Business Process Management (BPM 2017), pp 40-56

Ramos-Merino, M., Santos-Gago, J.M., Álvarez-Sabucedo, L.M., Alonso-Roris, V.M., \& Sanz-Valero, J. (2018). BPMN-E2: a BPMN extension for an enhanced workflow description. Software \& Systems Modeling, 1-21.

Ramos-Merino, M., Alvarez-Sabucedo, L.M., Santos-Gago, J.M., \& Sanz-Valero, J., (2018). “A BPMN Based Notation for the Representation of Workflows in Hospital Protocols", Journal of Medical Systems 42(10), 181.

Rekik, M., Boukadi, K., \& Ben-Abdallah, H. (2015) 'Specifying business process outsourcing requirements' in Proceedings of the 10th International Conference on Software Technologies (ICSOFT), Alsace, France, pp.175190.

Respicio, A., \& Domingos, D. 'Reliability of BPMN Business Processes', Volume 64, 2015, Pages 643-650

Rodríguez, A., Caro, A., Cappiello, C., Caballero, I.,' A BPMN Extension for Including Data Quality Requirements in Business Process Modeling', International Workshop on Business Process Modeling Notation (BPMN 2012), pp 116-125

Salles, G. B. M., Fantinato, M., Barros, V. A. and Albuquerque, Joao Porto de (2018). 'Evaluation of the StrAli-BPM approach: strategic alignment with BPM using agreements in different levels'. International Journal of Business Information Systems, 27 (4). pp. 433-465

Salnitri, M., Dalpiaz, F. \& Giorgini, P., 'Designing secure business processes with SecBPMN', Software \& Systems Modeling, 2017, Volume 16, Issue 3, pp 737-757 
Sang, K.S., \& Zhou B. 'BPMN Security Extensions for Healthcare Process', in 2015 IEEE International Conference on Computer and Information Technology; Ubiquitous Computing and Communications; Dependable, Autonomic and Secure Computing; Pervasive Intelligence and Computing, October 2015, Liverpool, UK

Stroppi, L.J.R., Chiotti, O., Villarreal, P.D.: Extending bpmn 2.0: Method and tool support. Business Process Model and Notation (2011) 59-73

Tranquillini, S., Daniel, F., Kucherbaev, P., \& Casati, F. (2015). 'Modeling, Enacting, and Integrating Custom Crowdsourcing Processes', Journal of ACM Transactions on the Web (TWEB), Volume 9, Issue 2, Article 7, pp. 7:17:43.

Vogel, J., Zobel, B., Jannaber, S., \& Thomas, O., (2018). “BPMN4SGA: A BPMN Extension for Smart Glasses Applications to enable Process Visualisations". INFORMATIK Workshop - Architectures, Processes, Security and Sustainability, 259-273, Bonn, Germany.

Yahya, F., Boukadi, K., Maamar, Z., \& Ben Abdallah, H. (2015). Enhancing Business Processes with Web 2.0 Features. International Conference on E-Business, Colmar, Alsace, France., 183-190, July 2015

Yahya, F., Boukadi, K., Maamar, Z., \& Ben-Abdallah, H. (2018), 'Social Business Process Modeling', In book: Encyclopedia of Information Science and Technology, Fourth Edition, Chapter 66, Publisher: IGI Global, pp. 765776

Yousfi, A., Bauer, C., Saidi, R., \& Dey, A.K. (2016). 'uBPMN: A BPMN extension for modeling ubiquitous business processes'. Information and Software Technology, Volume 74, pp. 55-68

Yousfi, A., Freitas, A.A., Dey, A.K., \& Saidi, R. (2015). 'The Use of Ubiquitous Computing for Business Process Improvement'. IEEE Transactions on Services Computing, 9, pp. 621-632.

Zaaboub Haddar, N., Makni, L., \& Ben Abdallah, H. 'Literature review of reuse in business process modeling'. Softw. Syst. Model. 13(3), 975-989 (2014)

Zerbato, F., Oliboni, B., \& Combi, C. 'BPMN-based Representation and Comparison of Clinical Pathways for Catheter-related Bloodstream Infections', in Proceedings of 2015 International Conference on Healthcare Informatics (ICHI), Dallas, Texas (USA), Oct. 2015 , 2015 , pp. 346-355

Zor, S., Schumm, D., Leymann, F., 2011. A Proposal of BPMN Extensions for the Manufacturing Domain, 44th CIRP International Conference on Manufacturing Systems. Madison, Wisconsin, USA. 